\title{
Neuropilin-1 mediates vascular permeability independently of vascular endothelial growth factor receptor-2 activation
}

\author{
Lise Roth $^{1,2}$, Claudia Prahst $^{1{ }^{\dagger}}$, Tina Ruckdeschel $^{1}$, Soniya Savant ${ }^{1, \ddagger}$, \\ Simone Weström ${ }^{1,++}$, Alessandro Fantin ${ }^{3}$, Maria Riedel ${ }^{1}$, \\ Mélanie Héroult ${ }^{1, \neq \ddagger}$, Christiana Ruhrberg ${ }^{3}$, and Hellmut G. Augustin ${ }^{1,2}$
}

${ }^{1}$ Division of Vascular Oncology and Metastasis, German Cancer Research Center (DKFZ-ZMBH Alliance), 69120, Heidelberg, Germany

${ }^{2}$ Department of Vascular Biology and Tumor Angiogenesis (CBTM), Medical Faculty Mannheim, Heidelberg University, 68167, Mannheim, Germany

${ }^{3} \mathrm{UCL}$ Institute of Ophthalmology, University College London, EC1V 9EL, London, UK

${ }^{\dagger}$ Current address: Center for Vascular Biology Research, Beth Israel Deaconess Medical Center, Boston, 02215, USA

${ }^{\ddagger}$ Current address: Dept. of Cell and Molecular Biology, Karolinska Institute, SE-171, Stockholm, Sweden

${ }^{+\dagger}$ Current address: Department of Medical Sciences, Uppsala Universitet, 751 05, Uppsala, Sweden

${ }^{\ddagger \ddagger}$ Current address: BAYER AG, Innovation Strategy Department, 51368, Leverkusen, Germany

Correspondence to:

Dr. Hellmut G. Augustin

Joint Research Division of Vascular Biology

Medical Faculty Mannheim (CBTM), Heidelberg University, German Cancer Research Center (DKFZ-ZMBH Alliance) Im Neuenheimer Feld 280, 69221 Heidelberg, Germany

Phone: +49-6221-421500

Email: augustin@angiogenese.de 


\begin{abstract}
Neuropilin-1 (NRP1) regulates developmental and pathological angiogenesis, arteriogenesis, and vascular permeability, acting as a coreceptor for Semaphorin 3A (Sema3A) and the 165-amino acid isoform of vascular endothelial growth factor A (VEGF-A $\left.A_{165}\right)$. NRP1 is also the receptor for the CendR peptides, a class of cell- and tissue-penetrating peptides with a specific $R-x-x-R$ C-terminal motif. Because the cytoplasmic domain of NRP1 lacks catalytic activity, NRP1 is mainly thought to act through the recruitment and binding to other receptors. We report here that the NRP1 intracellular domain mediates vascular permeability. Stimulation with VEGF- $\mathrm{A}_{165}$, a ligand-blocking antibody and a CendR peptide led to NRP1 accumulation at cell-cell contacts in endothelial cell monolayers, increased cellular permeability in vitro, and vascular leakage in vivo. Biochemical analyses, VEGF receptor 2 (VEGFR-2) silencing, and the use of a specific VEGFR blocker established that the effects induced by the CendR peptide and the antibody were independent of VEGFR-2. Moreover, leakage assays in mice expressing a mutant NRP1 lacking the cytoplasmic domain revealed that this domain was required for NRP1-induced vascular permeability in vivo. Hence, these data define a vascular permeability pathway mediated by NRP1 but independent of VEGFR-2 activation.
\end{abstract}




\section{Introduction}

Neuropilin-1 (NRP1) is a single-pass transmembrane receptor that is present in various tissues and cell types. First described as an orphan receptor with adhesion properties $(1,2)$, it was later identified as a receptor for members of the class 3 semaphorin (Sema3) family and of the Vascular Endothelial Growth Factor (VEGF) family (1-6). NRP1 is involved in multiple physiological and pathological processes. Its overexpression or targeted deletion in mice is embryonic lethal and results in severe cardiovascular defects, abnormal yolk sac and central nervous system vascularization, and defective great vessel remodeling $(4,7,8)$.

The extracellular domain of NRP1 is composed of different subdomains, which mediate its ligand binding specificity $(9,10)$. The transmembrane and the juxtamembrane domains are responsible for receptor dimerization, which is required for Sema3A and VEGF-A $\mathrm{A}_{165}$ signaling (11). The short NRP1 cytoplasmic tail lacks catalytic activity and interacts with the post synaptic density protein, Drosophila disc large tumor suppressor and zonula occludens-1 protein (PDZ) domain of the cytoplasmic protein GIPC1 (also known as synectin) (12). This interaction is essential for arteriogenesis because it directs the trafficking of endocytosed VEGF receptor 2 (VEGFR-2) into Rab5a-positive endosomes upon VEGF-A 165 stimulation (13). Due to its lack of catalytic activity, NRP1 is thought to act mainly as a binding element that recruits co-receptors responsible for intracellular signal transduction. For example, VEGF-A $\mathrm{A}_{165}$ binding to NRP1 induces the PDZ-binding domain-dependent formation of a NRP1-VEGFR-2 complex, which enhances VEGFR-2 intracellular signaling $(6,14,15)$.

VEGF is a potent permeability factor (16). Through binding to VEGFR-2, it triggers T-cell specific adaptor (TSAd)-mediated Src activation and focal adhesion kinase (FAK) phosphorylation (17-22). These events lead to VE-cadherin internalization, and ultimately to the loosening of adherens junctions with disruption of the endothelial barrier $(23,24)$. VEGF-mediated transcellular permeability has also been 
reported, and relies on vesiculo-vacuolar organelles (VVO), grape-like clusters of interconnected vesicles and vacuoles, which upon stimulation allow the passage of solutes and macromolecules (25).

A mutant form of VEGF-A $A_{165}$ that does not bind VEGFR-2 maintains its capacity to induce permeability, whereas VEGF-D binding and activation of VEGFR-2 is not sufficient to induce vascular leakage (26). These data suggest that other receptors participate in VEGF- $\mathrm{A}_{165}$-induced leakage. Several studies point to a role of NRP1 in this context: Selective NRP1 overexpression in porcine aortic endothelial cells demonstrates that it is required for VEGF- $A_{165}$-mediated permeability (27). Accordingly, a neutralizing NRP1 antibody attenuates vascular leakage upon VEGF-A $\mathrm{A}_{165}$ overexpression in mouse lungs (27). Furthermore, mice expressing a soluble form of NRP1 in the skin show reduced permeability through a mechanism that cannot be attributed solely to sequestration of VEGF- $A_{165}$, supporting a role for NRP1 in vascular permeability independent of VEGF-A $\mathrm{A}_{165}(28)$.

Another class of permeability-inducing NRP1 ligands is the CendR peptide family (29). The presence of a specific R-X-x-R motif at the C-terminal end of these peptides mediates NRP1 binding, followed by their rapid cell internalization and tissue penetration. CendR peptides induce the extravasation of associated cargo (such as nanoparticles) or co-injected drug into tissues, suggesting activation of a bulk transport system (30). Endocytosis of CendR peptides is distinct from previously known endocytic pathways, requires NRP1 interaction with synectin, and is regulated by nutrient supply to cells and tissues (31). However, the role of endocytosis in increased permeability and the mechanism through which CendR peptides exert their functions remain unclear.

In the present study, we identified the NRP1 cytoplasmic domain as essential for acute vascular hyperpermeability induced by different NRP1 ligands: a ligand-blocking antibody against NRP1 and a CendR peptide. We showed that these ligands induced NRP1 accumulation at endothelial cell-cell contacts, and triggered in vitro and in vivo permeability in a VEGFR-2-independent manner.

\section{Results}


VEGF-A 165 and a NRP1 ligand-blocking antibody induce NRP1 accumulation at endothelial cell-cell contacts

We investigated the role of NRP1 in VEGF-A 165 -induced vascular permeability by first studying NRP1 distribution in human umbilical vein endothelial cells (HUVECs) upon VEGF-A $A_{165}$ stimulation. NRP1, which was present in intracellular vesicles and dispersed at the plasma membrane (Fig. 1A; 0 min), strongly accumulated at cell-cell contacts within 5 min of VEGF-A $A_{165}$ stimulation (Fig. $1 \mathrm{~A} ; 5$ min). Over time, NRP1 was partially internalized (Fig. 1A; $30 \mathrm{~min}$ ). VEGF-A 165 induced a similar NRP1 intercellular accumulation in human dermal blood endothelial cells (HDBECS) (Sup. Fig. S1, upper panel). VEGF- $\mathrm{A}_{121}$, which has weaker affinity for NRP1 than VEGF-A $A_{165}$, but maintains high affinity for VEGFR-2 $(32,33)$, had no noticeable effect on NRP1 localization (Sup. Fig. 2, upper panel).

To examine whether direct binding of VEGF- $\mathrm{A}_{165}$ to NRP1 was responsible for NRP1 accumulation at cellcell contacts, we incubated HUVECs with a polyclonal blocking antibody directed against VEGF- $\mathrm{A}_{165}-\mathrm{NRP1}$

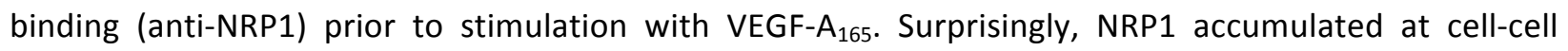
contacts after incubation with anti-NRP1 in the presence or even the absence of VEGF- $\mathrm{A}_{165}$, suggesting that this process occurred independently of VEGF-A $\mathrm{A}_{165}$ (Fig. 1B, lower panel, white arrows). The control IgG did not affect basal or VEGF-A $\mathrm{A}_{165}$-mediated NRP1 localization, confirming the specificity of the antibody-induced effect (Fig. 1B, upper panel). In HUVECs stimulated with anti-NRP1 alone, NRP1 initially accumulated at cell-cell contacts (Fig. 1C; 5 min) and partially internalized over time (Fig. 1C; 30 min). Hence, anti-NRP1 did not act as an antagonist of NRP1 intercellular accumulation, but as an agonist. Similar results were observed in HDBECs (Sup. Fig. S1, middle panel).

\section{A tetrameric CendR peptide induces NRP1 accumulation at endothelial cell-cell contacts}

Since both VEGF-A $A_{165}$ and anti-NRP1 induced NRP1 relocalization in endothelial monolayers, we hypothesized that other NRP1 ligands might have a similar effect. We used the prototypic CendR peptide RPARPAR, which, like VEGF- $A_{165}$, binds to the NRP1 b1 binding pocket (34). Stimulation of 
HUVECs with monomeric RPARPAR did not affect NRP1 localization (Sup. Fig. 2, lower panel). Since CendR peptides are more efficient at penetrating tissues in oligomeric form (29), we conjugated biotinylated RPARPAR to neutravidin (NA) to obtain a tetrameric peptide (NA-RPARPAR). NA-RPARPAR induced a strong and rapid NRP1 accumulation at cell-cell contacts, which persisted for longer periods compared to VEGF-A $A_{165}$ and anti-NRP1 stimulation (Fig. 1D). A similar relocalization was observed in HDBECs (Sup. Fig. S1, lower panel). These results confirmed that NRP1 intercellular accumulation was not VEGF-A $\mathrm{A}_{165}$-specific, and suggested that ligand-induced receptor clustering was necessary to promote NRP1 relocalization. Sema3A primarily binds the NRP1 a1-a2 domains, and can also bind the b1 domain through its basic C-terminal extremity $(9,10,35-37)$. At concentrations up to $250 \mathrm{ng} / \mathrm{ml}$, it did not cause NRP1 accumulation at cell-cell contacts in HUVEC monolayers (Sup. Fig. S3). Nevertheless, at 500 and $1000 \mathrm{ng} / \mathrm{ml}$, Sema3A induced a faint and partial NRP1 relocalization after 30 and 5 min incubation, respectively (Sup. Fig. S3). These results suggest that a strong affinity binding to NRP1 b1 domain is required to trigger receptor relocalization.

NRP2, another member of the NRP family, shares a strong sequence and structural homology with NRP1. It binds to VEGF family members as well as to CendR peptides $(38,39)$. Staining for NRP2 in stimulated HUVEC monolayers revealed that VEGF- $\mathrm{A}_{165}$ had no effect on NRP2 localization, whereas NARPARPAR induced NRP2 accumulation at cell-cell contacts, albeit to a lesser degree compared to NRP1 (Sup. Fig. S4). These results are in accordance with the lower binding affinity of VEGF-A $A_{165}$ and CendR peptides for NRP2 compared to NRP1 $(33,39)$.

NRP1 relocalization does not occur through an increase in NRP1 cell surface abundance

To elucidate whether an increase of NRP1 cell surface abundance was responsible for its accumulation at cell-cell contacts, we first determined global NRP1 abundance in HUVECs before and after stimulation with VEGF-A $A_{165}$, anti-NRP1 and NA-RPARPAR. Neither NRP1 mRNA nor NRP1 protein abundance were changed after 5 min stimulation (Sup. Fig. S5A, B). We next confirmed that the ligand-blocking antibody 
anti-NRP1 could not access intracellular NRP1 pools by directly staining non-permeabilized HUVEC monolayers, indicating that it triggered NRP1 relocalization by directly engaging cell-surface NRP1 (Sup. Fig. S5C). We also considered whether ligand binding to surface NRP1 could induce the recruitment of cytoplasmic NRP1 to the plasma membrane, and performed flow cytometry analyses to determine NRP1 surface abundance on HUVECs before and after stimulation. Each of the three examined ligands induced a unique response (Sup. Fig. S5D). VEGF-A $A_{165}$ stimulation triggered an increase in NRP1 cell surface abundance at 2 and $5 \mathrm{~min}$, followed by a decrease at 30 and $60 \mathrm{~min}$, while anti-NRP1 and NA-RPARPAR initially induced no change or a decrease in NRP1 cell surface abundance, respectively (Sup. Fig. S5D). This difference might be due to the specificity of VEGF- $A_{165}$, which also binds VEGFR-2 on HUVECs. Thus, the results suggest that NRP1 relocalization at cell-cell contacts did not require an increase of NRP1 cell surface abundance. Accordingly, pre-incubation of HUVEC monolayers with nocodazole, to block possible microtubule-mediated NRP1 transport to the membrane (40-42), had no effect on NRP1 relocalization (Sup. Fig. 5E). Taken together, the data imply that NRP1 relocalizes laterally through the plasma membrane.

VEGF- $A_{165}, N A-R P A R P A R$ and anti-NRP1 induce endothelial leakage in vitro and in vivo We further investigated NRP1 relocalization at cell-cell contacts by co-staining HUVECs with VE-cadherin (Fig. 2A). Upon stimulation with VEGF-A ${ }_{165}$, NA-RPARPAR or anti-NRP1, NRP1 was found in close proximity or co-localized (white arrows) with VE-cadherin (Fig. 2A). We hypothesized that the NRP1 that accumulated at endothelial junctions participated in vascular permeability, and we consequently studied the effect of NRP1 stimulation on FITC-dextran leakage through a HUVEC monolayer. After 5 min stimulation, VEGF-A 165 , NA-RPARPAR and anti-NRP1 induced a significant increase of dye leakage through the endothelial monolayer compared to PBS (Fig. 2B). A time-course experiment in which fluorescence leakage was measured after 5, 15 and 30 min stimulation confirmed that the maximum leakage induced by the ligands compared to PBS occurred after 5 min (Sup. Fig. S6A). Monomeric 
RPARPAR did not significantly affect endothelial leakage (Sup. Fig. S6B), in line with its inability to induce NRP1 relocalization (Sup. Fig. 2).

We next performed Miles leakage assays to measure permeability in vivo. VEGF-A $\mathrm{A}_{165}$ and NA-RPARPAR significantly increased Evans Blue extravasation in the skin compared to PBS, as previously shown (29, 43) (Fig. 2C). Furthermore, we showed that anti-NRP1 also significantly induced vascular leakage (Fig. 2C). Taken together, our data correlate ligand-mediated NRP1 accumulation at cell-cell junctions in endothelial monolayers with induced in vitro and in vivo vascular leakage, supporting a role for NRP1relocalization in vascular permeability.

NA-RPARPAR and anti-NRP1 act through a signaling pathway distinct from VEGF-A $A_{165}$ Binding of VEGF-A $A_{165}$ to NRP1 bridges VEGFR-2 and NRP1 and enhances VEGFR-2 signaling (14). We therefore asked if NA-RPARPAR and anti-NRP1 would similarly induce VEGFR-2 activation, and if this activation was required for NRP1 intercellular accumulation and vascular leakage. Towards this end, we analyzed the phosphorylation status of VEGFR-2 upon stimulation with the three different ligands. Whereas VEGF-A $\mathrm{A}_{165}$ induced strong VEGFR-2 phosphorylation, neither NA-RPARPAR nor anti-NRP1 activated the receptor (Fig. 3A). Moreover, the phosphorylation status of the VEGFR-2 downstream kinases Akt, p38 and extracellular signal-regulated kinase 1 and 2 (ERK1/2), which are involved in VEGF$A_{165}$-induced permeability $(44,45)$, did not increase in HUVECs stimulated with NA-RPARPAR or antiNRP1 compared to unstimulated cells (Fig. 3B). These results were confirmed in HDBEC monolayers (Sup. Fig. S7A, B), and demonstrated that NA-RPARPAR and anti-NRP1-induced effects were not mediated by VEGFR-2 activation.

VEGF- $\mathrm{A}_{165}$-induced destabilization of the endothelial barrier is mediated by Src and FAK activation (19, 20, 22). We consequently quantified kinase phosphorylation following stimulation by the different ligands. VEGF- $\mathrm{A}_{165}$ induced a small but significant increase in the phosphorylation of $\mathrm{Tyr}^{416}$ in $\mathrm{Src}$ at 5, 15 and $30 \mathrm{~min}$, and in the phosphorylation of $\mathrm{Tyr}^{397}$ in FAK at 15 and $30 \mathrm{~min}$ (Fig. 3C, D, E). NA-RPARPAR and 
anti-NRP1 did not alter FAK Tyr ${ }^{397}$ phosphorylation in HUVECs (Fig. 3C, E), or in HDBEC monolayers (Sup. Fig. S7C, D). However, they both triggered a transient increase in the phosphorylation of $\mathrm{Tyr}^{416}$ in SrC (Fig. 3C, D). To further study the role of Src activation in ligand-induced permeability, we performed Miles assays after systemic Src inhibition by the Src and Abl inhibitor SKI-606 (bosutinib). As previously shown (46), SKI-606 significantly inhibited VEGF- $\mathrm{A}_{165}$-induced vascular leakage. However, despite a decrease of the mean leakage values, no significant vascular permeability inhibition was observed for NA-RPARPAR and anti-NRP1 (Sup. Fig. S8A-C). Therefore, even though NA-RPARPAR and anti-NRP1 transiently activated Src in endothelial monolayers, this activation was not necessary for downstream vascular leakage.

Accumulation of NRP1 at cell-cell contacts and subsequent vascular leakage are independent of the activation of the tyrosine kinase activity of VEGFR

We next examined the consequences of inhibiting the tyrosine kinase activity of VEGFRs on NRP1 relocalization and vascular leakage. The small tyrosine kinase inhibitor PTK787/ZK222584 (PTK/ZK, Vatalanib) targets VEGFR-1, VEGFR-2 and VEGFR-3 (IC 50 of $0.077,0.037$ and $0.27 \mu \mathrm{M}$, respectively), and to a lesser extent platelet-derived growth factor receptor and c-kit (47). We incubated cultured HUVEC monolayers with PTK/ZK prior to stimulation with the different ligands. As expected, PTK/ZK inhibited VEGF-A 165 -induced VEGFR-2 phosphorylation (Fig. 4A). However NRP1 accumulation at cell-cell contacts induced by VEGF- $\mathrm{A}_{165}$, anti-NRP1 and NA-RPARPAR was not affected by PTK/ZK treatment, showing that this process did not require VEGFR-2 activation (Fig. 4B). Futhermore, knockdown of VEGFR-2 in HUVECs (Fig. 4C) did not affect NRP1 relocalization at cell-cell contacts (Fig. 4D). These data demonstrated that

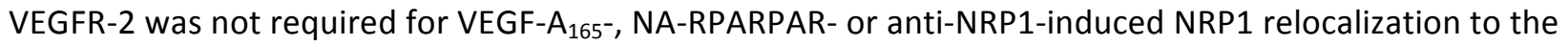
endothelial junctions.

For in vivo analysis, we performed Miles assay experiments after systemic circulation of PTK/ZK. The kinase inhibitor did not significantly affect VEGF-A ${ }_{165^{-}}$, NA-RPARPAR- or anti-NRP1-induced vascular 
leakage, showing that these ligands induced permeability independently of VEGFR activation (Fig. 4E, F, G). Yet, PTK/ZK significantly inhibited VEGF-A 121 -induced leakage (Fig. $4 \mathrm{H}$ ), demonstrating the efficiency of the inhibitor at the concentration used, and suggesting that this VEGF-A isoform required VEGFR activation to induce permeability.

\section{NRP1 cytoplasmic domain mediates NRP1 accumulation at cell-cell contacts and vascular permeability}

We next asked if the NRP1 cytoplasmic domain could directly mediate VEGF- $\mathrm{A}_{165^{-}}$, NA-RPARPAR- and anti-NRP1-induced effects. We overexpressed NRP1 in HUVECs as full-length NRP1 or as truncated NRP1 lacking the intracellular domain (NRP1 $\triangle \mathrm{C}$ ), and stimulated the cells with different concentrations of VEGF-A $\mathrm{A}_{165}$ for 5 min. GFP-transfected cells (GFP) were used as a control. VEGF-A $\mathrm{A}_{165}$ triggered VEGFR-2 phosphorylation in a dose-dependent manner in GFP-, NRP1-, and NRP1 $\triangle$ C-transfected cells, showing that VEGFR-2 signaling pathway was functional (Fig. 5A). However, whereas full-length NRP1 accumulated at cell-cell contacts upon VEGF-A $\mathrm{A}_{165}$ stimulation (Fig. 5B, white arrows), NRP1 $\Delta C$ distribution remained unchanged (Fig. 5B). Hence, NRP1 cytoplasmic domain was required for VEGF$\mathrm{A}_{165}$-induced NRP1 accumulation at the endothelial contacts.

To test whether NRP1 cytoplasmic domain also mediated permeability in vivo, we performed Miles assay experiments in mice lacking the cytoplasmic domain of NRP1 (NRP1cyto ${ }^{-1}$ ). These mice are viable and fertile, with no overt developmental defects, suggesting that the extracellular domain mediates NRP1 functions during angiogenesis (48). We observed a decrease in the VEGF- $\mathrm{A}_{165}$-induced leakage in

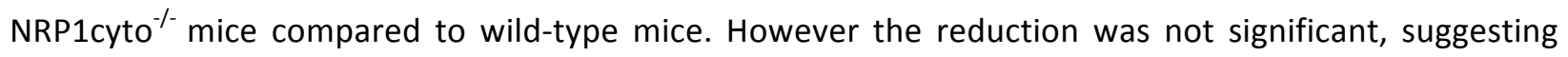
that the NRP1 cytoplasmic domain could participate in VEGF-A $\mathrm{A}_{165}$-induced vascular leakage despite not being necessary (Fig. 5C). On the other hand, vascular leakage was significantly decreased in NRP1cyto-/mice compared to their wild-type littermates upon intradermal injection of either NA-RPARPAR or antiNRP1, establishing that these non-VEGFR-2 binding NRP-1 ligands induced vascular extravasation through the NRP1 cytoplasmic domain (Fig. 5D, E). 


\section{Discussion}

In this study, we have identified direct ligand binding to NRP1 as a VEGFR-2-independent mechanism to induce vascular permeability in vitro and in vivo. Three different NRP1 ligands induced its accumulation at endothelial cell-cell contacts and enhanced solute permeability in a process that required the NRP1 cytoplasmic domain. Upon stimulation with VEGF-A $A_{165}$, anti-NRP1 and NA-RPARPAR, NRP1 rapidly accumulated at intercellular contacts of HUVEC or HDBEC monolayers, co-localized or was in close proximity with VE-cadherin. This fast NRP1 concentration at endothelial junctions corresponds with a role of the receptor in acute hyperpermeability and could constitute the first step in junction opening or VVO formation. NRP1 relocalization upon ligand stimulation did not require an increase of NRP1 surface abundance and was not affected by inhibition of microtubule transport, suggesting that the receptor relocalized laterally across the plasma membrane to accumulate at intercellular junctions. Moreover, the data suggest that ligands that induce NRP1 oligomerization trigger its relocalization. NRP1 dimerizes through its juxtamembrane and transmembrane domains (9-11), and a peptide mimicking its transmembrane domain successfully inhibits VEGF-A 165- $^{-}$and Sema3A-induced NRP1-mediated effects $(11,49)$. The findings showing that monomeric RPARPAR did not affect NRP1 localization patterns and did not increase FITC-dextran leakage through HUVEC monolayers, together with a previous study demonstrating the increased NA-RPARPAR efficiency in vivo compared to RPARPAR (29), support the concept that NRP1 multimerization is also required for cell-cell contact accumulation and permeability induction.

VEGF-A 165 and NA-RPARPAR share the same binding site within the NRP1 b1 binding pocket $(34,37)$. However, NRP1 binding to the b1 domain appears not to be sufficient to induce NRP1 cell-cell contacts accumulation. VEGF-A $\mathrm{A}_{121}$, which binds to b1 but lacks exon 7 present in other VEGF-A isoforms, did not induce NRP1 relocalization. Likewise, VEGF- $\mathrm{A}_{121}$-induced leakage was inhibited by PTK/ZK in the Miles 
assay, suggesting that this VEGF-A isoform acts primarily through the tyrosine kinase activity of VEGFRs to induce permeability. Sema3A is another vascular permeability-inducing factor that requires NRP1 for inducing leakage (46). NRP1 a1 and a2 domains constitute the class 3 semaphorin primary binding sites, and whether Sema3 can compete with VEGF- $\mathrm{A}_{165}$ for binding to the b1 domain is still under debate (5053). In our hands, Sema3A only triggered a weak NRP1 relocalization at cell-cell contacts when used at high concentrations, and thus probably induces vascular leakage through a different mechanism than VEGF-A 165, NA-RPARPAR and anti-NRP1. These data support the notion that not all NRP1 ligands trigger permeability through the same mechanism, which could reflect different physiological settings.

Unlike VEGF-A $A_{165}$ and NA-RPARPAR, which bind to a specific site in NRP1, anti-NRP1 is a polyclonal antibody generated with an antigen covering a large portion of the receptor. Similar to VEGF-A $\mathrm{A}_{165}$ and NA-RPARPAR, it induced NRP1 accumulation at endothelial cell-cell contacts in vitro and vascular leakage in vivo. Strikingly, the same antibody has been previously used to inhibit CendR peptide-induced vascular leakage by antibody administration 30 min prior to Evans Blue injection (29). In vivo, leakage induced by a single exposure of permeability-inducing agent begins within a minute and lasts for maximally $30 \mathrm{~min}$ (54). Therefore, in the aforementioned work, the antibody permeability-inducing effects might have ended by the time the dye was injected. Another study used a ligand-blocking antiNRP1 antibody to inhibit leakage in vitro and in vivo in a lung wet-dry weight model (27). This antibody was produced with an uncharacterized antigen, and might therefore bind to different site(s) than the antibody used in the present work. Moreover, the organ studied (lung compared to skin), the route (intraperitoneal compared to intradermal) and frequency (multiple doses compared to single dose) of administration, the quantities used and the length of treatment before stimulation were significantly different between these and our study, and the effects observed can therefore not be formally compared. 
NRP1 has a closely related family member, NRP2. NRP1 and NRP2 share common ligands, among them VEGF-A $A_{165}$ and CendR peptides $(38,39)$. However, HUVEC stimulation with VEGF- $A_{165}$ had no effect on NRP2 localization, whereas NA-RPARPAR-induced NRP2 accumulation at the cell-cell contacts was weaker than the one observed with NRP1. These data are in accordance with VEGF-A $\mathrm{A}_{165}$ having lower affinity for NRP2 ( $\mathrm{k}_{\mathrm{D}}=150 \mathrm{nM}$ compared to $3 \mathrm{nM}$ for NRP1, (33)), and with the lower binding efficacy of phage-bound CendR peptides towards NRP2 compared to NRP1 (39). Nevertheless, our data unambiguously demonstrate that NRP2 is also able to relocalize upon stimulation. NRP1 and NRP2 exhibit a differential distribution pattern, and NRP2 predominates in lymphatic vessels in particular (55). VEGF-C is a high affinity NRP2 ligand and a potent permeability factor involved in lymphangiogenesis $(56,57)$. Similar to NRP1, NRP2 could therefore participate in vascular extravasation upon VEGF-C stimulation.

As a ligand for VEGF-A $\mathrm{A}_{165}$, NRP1 is generally viewed as an enhancer of VEGFR-2-induced signaling (14). However, we found that neither NA-RPARPAR nor anti-NRP1 triggered VEGFR-2 phosphorylation. Furthermore, VEGFR-2 knockdown did not affect NRP1 relocalization in HUVEC monolayers, and the multi-kinase inhibitor PTK/ZK similarly did not prevent NRP1 accumulation at cell-cell contacts, showing that VEGFR-2 was not required for this process. Moreover, vascular leakage induced by these ligands was not inhibited by PTK/ZK, further demonstrating that VEGFR-2 inhibition does not affect VEGF- $\mathrm{A}_{165}$, NA-RPARPAR and anti-NRP1-induced vascular leakage. Using mice lacking the NRP1 cytoplasmic domain, we demonstrated the direct involvement of this domain in NA-RPARPAR and anti-NRP1-induced permeability. Hence, both ligands induce vascular leakage in a VEGFR-2-independent manner through NRP1 cytoplasmic domain. These data are in line with previous publications showing a direct role for NRP1 in endothelial cell migration, survival or permeability independently of VEGFR-2 $(27,58,59)$. They furthermore agree with the observation that CendR peptides require NRP1 cytoplasmic domain to induce internalization (31). However, VEGF-A $\mathrm{A}_{165}$-mediated permeability was not significantly inhibited in 
NRP1cyto $^{-/}$mice. VEGF-A $\mathrm{A}_{165}$ is therefore able to compensate for the loss of NRP1 cytoplasmic domain, presumably through VEGFR-2. Reciprocally, PTK/ZK did not significantly decrease VEGF- $\mathrm{A}_{165}$-mediated permeability, showing that VEGF-A $A_{165}$ can circumvent VEGF receptor inhibition. VEGF- $A_{165}$ therefore seems capable of inducing vascular leakage through both VEGFR-2 and NRP1, whereas NA-RPARPAR and anti-NRP1 only act through NRP1 (Fig. 5F). This duality could account for the high variability observed in response to VEGF-A $\mathrm{A}_{165}$ in the Miles assay. Whether VEGFR-2 and NRP1 act as a complex as shown previously remains to be determined.

The downstream events linking NRP1 cytoplasmic domain and vascular leakage are still unclear. NARPARPAR and anti-NRP1 did not activate the VEGFR-2 downstream kinases Akt, p38 and ERK1/2. A moderate and transient activation of Src upon NA-RPARPAR and anti-NRP1 stimulation was observed, suggesting that NRP1 activation could lead to Src-mediated endothelial destabilization and paracellular permeability independently of VEGFR-2 (24). However, in vivo inhibition of Src did not prevent NARPARPAR and anti-NRP1-induced vascular leakage. Moreover, FAK was not significantly activated following anti-NRP1 or NA-RPARPAR binding $(20,22)$. The involvement of FAK/Src kinases in NRP1mediated permeability therefore seems accessory. A recent study has demonstrated the importance of the NRP1-synectin interaction in CendR peptide-mediated endocytosis (31), and synectin could similarly be involved in CendR peptide-mediated permeability. Moreover, the authors of that study reported the formation of engulfment structures in cancer cells that structurally resemble macropinocytotic vesicles. They proposed that these structures represent the first step in the formation of VVO, which would induce transcellular permeability (25). It is therefore tempting to hypothesize that vascular leakage induced by NRP1-ligand binding could result from a transcellular mechanism. A better comprehension of the physiological and/or pathological context in which NRP1 mediated-permeability takes place will help identifying the route mediating leakage, and could offer new perspectives in the treatment of acute or chronic hyperpermeability. 


\section{Materials and Methods}

\section{Antibodies and reagents}

For immunofluorescence, NRP1 was stained with the monoclonal mouse anti-NRP1 antibody (Miltenyi Biotech), NRP2 with the polyclonal rabbit anti-NRP2 and VE-cadherin with the polyclonal goat anti-VEcadherin (Santa Cruz Biotechnology). The secondaries anti-mouse Cy3, anti-rabbit CY3 (Dianova), antisheep A488 and anti-sheep 546 (Life Technologies) were used. Nuclei were stained with Hoechst (Sigma Aldrich). The mouse monoclonal VioBright FITC anti-NRP1 antibody (Miltenyi) and a mouse isotype control (Abcam) were used for flow cytometry. For Western blots, the rabbit monoclonal anti-VEGFR-2, mouse monoclonal anti-phospho-p44/42 MAPK $\mathrm{Thr}^{202} / \mathrm{Tyr}^{204}$, rabbit monoclonal anti-phospho-Akt $\mathrm{Ser}^{473}$, rabbit polyclonal anti-Akt, rabbit monoclonal anti-phospho-p38 $\mathrm{Thr}^{180} / \mathrm{Tyr}^{182}$, rabbit monoclonal anti-p38, rabbit polyclonal anti-phospho $\mathrm{Src}_{\mathrm{Tyr}}{ }^{416}$, mouse monoclonal anti-Src, and rabbit monoclonal anti-phospho-FAK Tyr $^{397}$ were purchased from Cell Signaling Technology. Rabbit polyclonal anti-ERK1/2, rabbit polyclonal anti-FAK, goat polyclonal anti-NRP1, goat polyclonal anti-actin and mouse monoclonal anti-GAPDH were from Santa Cruz Biotechnology. The mouse monoclonal anti-phosphotyrosine was from Millipore and the mouse monoclonal anti-tubulin from Sigma. For immunoprecipitation, the mouse monoclonal anti-VEGFR-2 was from Reliatech.

The ligand-blocking sheep anti-human NRP1 and goat anti-rat NRP1 antibodies were purchased from R\&D Systems. Sheep and goat IgG (Dianova) were used as controls. The recombinant purified proteins mouse VEGF- $A_{120}$, mouse VEGF- $A_{164}$ and human Sema3A were purchased from R\&D Systems, and human VEGF-A $\mathrm{A}_{165}$ and human VEGF-A $\mathrm{A}_{121}$ from Reliatech. Biotin-RPARPAR was synthetized by Peptide Specialty Laboratories and tetrameric RPARPAR was obtained by conjugation with neutravidin (Pierce) as described previously (39). The inhibitor PTK787/ZK222584 was purchased from Cell Signaling Technology, SKI-606 from Abcam, and nocodazole from Sigma. 


\section{Cell culture}

HUVECs and HDBECs were purchased from Promocell. HUVECs were cultured in Endopan 3 medium completed with the corresponding supplements (PAN Biotech), and HDBECs in Endothelial Cell Growth Medium MV2 completed with the corresponding supplement mix (Promocell). Cells were used between passages 1 and 7 and cultured at $37^{\circ} \mathrm{C}, 5 \% \mathrm{CO} 2$.

\section{Cell transfection}

Adenoviral constructs containing the cDNA for GFP, NRP1 and NRP1 $\triangle$ C were generated with the Gateway System (Life Technologies) according to the manufacturer's instructions. HUVECs were transfected with 100 ifu/cell one day before stimulation. For gene silencing experiments, HUVECs were transfected with $100 \mathrm{nM}$ KDR siRNA (s7822 and s7823) or non-coding siRNA (Life Technologies) using 6 $\mu$ Oligofectamin in Opti-MEM+ GlutaMAX-I (Life Technologies). Validation of VEGFR-2 down-regulation by quantitative real-time PCR (qRT-PCR) analysis and functional experiments were performed $48 \mathrm{~h}$ posttransfection.

\section{Cell stimulation and Immunostaining}

HUVECs were grown on $0.2 \%$ gelatin-coated coverslips (Thermo Scientific) until they formed a tight monolayer. After starvation with Endopan 3 without supplement for 5-6 h, they were stimulated with VEGF-A $165(50 \mathrm{ng} / \mathrm{ml})$, NA-RPARPAR (peptide concentration: $1 \mu \mathrm{M})$, anti-NRP1 $(3 \mu \mathrm{g} / \mathrm{ml})$, or otherwise specified ligand. Cells were fixed and permeabilized with ice-cold methanol/acetone (50/50), blocked with PBS/BSA $3 \%$, and stained with primary antibodies at $4{ }^{\circ} \mathrm{C}$ overnight. The appropriate secondary antibodies were incubated for $1 \mathrm{~h}$ at room temperature. Nuclei were counterstained with Hoechst. The coverslips were mounted in Fluoromount G mounting medium (eBioscience). Stainings were acquired on a motorized inverted Observer.Z1 (Zeiss) or a Zeiss Ism 710 confocal microscope. At least 3 microscopic 
fields were analyzed per monolayer. With on average 20 cells per microscopic field of view, at least 150 cells were analyzed per experiment.

To inhibit VEGF- $\mathrm{A}_{165}-\mathrm{NRP1}$ binding, ligand-blocking anti-NRP1 or its control sheep IgG were added to the starved monolayers at $4{ }^{\circ} \mathrm{C} 30$ min before stimulation with VEGF- $A_{165}$. PTK/ZK (100 nM) and nocodazole $(20 \mu \mathrm{M})$ or the corresponding DMSO controls were added at $37^{\circ} \mathrm{C} 30 \mathrm{~min}$ prior to stimulation.

\section{Flow cytometry}

NRP1 surface expression in HUVECs before and after stimulation was analyzed by flow cytometry. Single cell suspensions were incubated with conjugated mouse anti-NRP1 or with the corresponding isotype control in basal medium containing $1 \%$ FCS for 30 min on ice. Samples acquisition was performed with a BD FACS Canto II flow cytometer and subsequent analysis was done using FlowJo software.

In vitro permeability assay

HUVECs were grown on $0.4 \mu \mathrm{m}$ fibronectin-coated (R\&D Systems) Transwell filters (Corning Incorporated Coaster Transwell). After 48 h, cells were starved with Endopan 3 without supplement for 5-6 h. The medium in the upper well was then replaced by FITC-dextran $70 \mathrm{kDa}(0.5 \mathrm{mg} / \mathrm{ml}$ in PBS, Sigma). Cells were stimulated in the lower well with PBS containing VEGF- $A_{165}(50 \mathrm{ng} / \mathrm{ml})$, NA-RPARPAR (peptide concentration: $1 \mu \mathrm{M})$, RPARPAR $(1 \mu \mathrm{M})$ or anti-NRP1 $(3 \mu \mathrm{g} / \mathrm{ml})$. The fluorescence in the lower well was read at $520 \mathrm{~nm}$.

\section{Immunoprecipitation and Western blots}

HUVECs were grown on $0.2 \%$ gelatin-coated dishes until they formed a tight monolayer. After starvation with Endopan 3 without supplement, cells were stimulated with VEGF-A 165 (50 ng/ml), NARPARPAR (peptide concentration: $1 \mu \mathrm{M})$ or anti-NRP1 $(3 \mu \mathrm{g} / \mathrm{ml})$. Cells were washed with ice-cold PBS containing $1 \mathrm{mM}$ orthovanadate, and lysed in a Tris/NaCl buffer $(25 \mathrm{mM} / 150 \mathrm{mM})$ containing $5 \mathrm{mM}$ EDTA, $1 \% \mathrm{NP}-40,100 \mathrm{mM} \mathrm{NaF}$ and $10 \mathrm{mM} \mathrm{Na} 4 \mathrm{P} 2 \mathrm{O} 7$ in presence of $1 \mathrm{mM}$ orthovanadate and of 
proteinase inhibitor mix G (SERVA electrophoresis). For immunoprecipitation, cell lysates were incubated overnight with $1 \mu \mathrm{g}$ anti-VEGFR-2 and protein-G sepharose (GE Healthcare). The sepharose beads were washed and the samples were boiled in sample buffer $(250 \mathrm{mM}$ Tris/ $\mathrm{HCl}$ pH 6.8, 10\% SDS, $0.5 \%$ bromophenol blue, $50 \%$ glycerin, supplemented with $10 \% \beta$-mercaptoethanol). Samples were subjected to $8 \%$ SDS-PAGE, blotted onto a MeOH-activated PVDF membrane, and probed with antibodies directed against VEGFR-2 and phosphotyrosine. To assess the phosphorylation of ERK1/2, Akt, p38, Src and FAK, total protein lysates were heated to $95^{\circ} \mathrm{C}$ in sample buffer, subjected to $10 \%$ SDSPAGE and blotted onto a PVDF membrane probed with the desired antibodies. Western blot analysis was performed using horseradish peroxidase-conjugated secondary antibodies (DAKO) and bound antibody was visualized using ECL (Pierce). To reprobe blots, membranes were stripped in a stripping solution (Millipore) for 7-10 $\mathrm{min}$, and reprobed with the desired antibodies. When indicated, the resultant intensities were quantified using Fiji Is Just ImageJ (Fiji) software.

\section{Quantitative real-time PCR}

RNA of HUVECs was isolated with RNeasy Mini Kit (Qiagen). cDNA was synthesized with QuantiTect Reverse Transcription Kit (Qiagen). Subsequent qRT-PCR was performed with TaqMan Fast Advanced Mastermix (Life Technologies) and the desired Taqman probes (GAPDH: Hs02758991_g1; ACTB: Hs01060665_g1; KDR: Hs00911700_m1; NRP1: Hs00826128_m1, Life Technologies) with a Roche Light Cycler 480.

\section{Miles assay}

Evans Blue (100 $\mu \mathrm{l} ; 1 \%$ in sterile saline; Sigma Aldrich) was systemically injected in the lateral tail vein of C57BL/6 mice, wild-type or knock-out for the NRP1 cytoplasmic domain (NRP1cyto ${ }^{-/},(40)$ ). After 15 min circulation, $20 \mu \mathrm{l}$ of ligand-containing solution and its control counterpart were injected each in one footpad of the mouse, which allowed having an internal control for each mouse (PBS compared to $\mathrm{NaCl}$, 
VEGF-A 164 or VEGF-A $\mathrm{A}_{120}(50 \mathrm{ng})$ compared to $0.9 \% \mathrm{NaCl}$; anti-NRP1 antibody $(5 \mu \mathrm{g})$ compared to goat IgG; NA-RPARPAR (peptide concentration: $30 \mu \mathrm{M}$ ) compared to neutravidin). Alternatively, the ligandcontaining solution was injected intradermally in the abdomen region (Fig 5E). After 15 min, the skin containing the extravasated dye was excised with a $4 \mathrm{~mm}$ punch (Stiefel). Evans Blue was extracted by incubation in formamide at $55{ }^{\circ} \mathrm{C}$ overnight, and quantified by spectrometry at $620 \mathrm{~nm}$ (Thermo Scientific Multiskan EX). Results are expressed as a ratio between the ligand-induced absorbance (in one foot) and its corresponding control-induced absorbance (in the other foot of the same mouse). When needed, $10 \mathrm{mg} / \mathrm{kg}$ PTK/ZK, $5 \mathrm{mg} / \mathrm{kg}$ SKI-606 or the corresponding DMSO controls were injected intraperitoneally $30 \mathrm{~min}$ prior to Evans Blue injection. Animals were handled in accordance with the guidelines of the Regierungspräsidium Karlsruhe, Germany (Permit N G65-15).

\section{Statistical analysis}

Data were analyzed with GraphPad Prism (GraphPad Software Inc). The statistical tests applied are indicated in the figure legend for each experiment. 


\section{Supplementary Materials}

Fig. S1. VEGF-A $A_{165}$, the ligand-blocking anti-NRP1 antibody, and NA-RPARPAR induce NRP1 accumulation at cell-cell contacts in HDBECs.

Fig. S2. VEGF-A $A_{121}$ and monomeric RPARPAR do not induce NRP1 accumulation at endothelial cell-cell contacts.

Fig. S3. High concentrations of Sema3A induce weak NRP1 accumulation at cell-cell contacts.

Fig. S4. NRP2 accumulates at cell-cell contacts upon stimulation with NA-RPARPAR, but not with VEGF$\mathrm{A}_{165}$.

Fig. S5. NRP1 relocalizes at cell-cell contacts laterally through the plasma membrane.

Fig. S6. Maximum endothelial leakage is observed after $5 \mathrm{~min}$, and monomeric RPARPAR does not induce endothelial leakage.

Fig. S7. NA-RPARPAR and anti-NRP1 do not activate VEGFR-2, Akt, ERK1/2, p38, and FAK in HDBECs.

Fig. S8. The Src inhibitor SKI-606 does not inhibit NA-RPARPAR- and anti-NRP1-induced vascular leakage. 


\section{References and Notes}

1. S. Takagi, T. Tsuji, T. Amagai, T. Takamatsu, H. Fujisawa, Specific cell surface labels in the visual centers of Xenopus laevis tadpole identified using monoclonal antibodies. Dev. Biol. 122, 90-100 (1987).

2. Z. He, M. Tessier-Lavigne, Neuropilin is a receptor for the axonal chemorepellent semaphorin III. Cell 90, 739-751 (1997).

3. S. Takagi, Y. Kasuya, M. Shimizu, T. Matsuura, M. Tsuboi, A. Kawakami, H. Fujisawa, Expression of a cell adhesion molecule, neuropilin, in the developing chick nervous system. Dev. Biol. 170, 207-222 (1995).

4. T. Kitsukawa, M. Shimizu, M. Sanbo, T. Hirata, M. Taniguchi, Y. Bekku, T. Yagi, H. Fujisawa, Neuropilin-Semaphorin III/D-mediated chemorepulsive signals play a crucial role in peripheral nerve projection in mice. Neuron 19, 995-1005 (1997).

5. A. Kolodkin, D. V. Levengood, E. G. Rowe, Y. T. Tai, R. J. Giger, D. D. Ginty, Neuropilin is a semaphorin III receptor. Cell 90, 753-762 (1997).

6. S. Soker, S. Takashima, H. Q. Miao, G. Neufeld, M. Klagsbrun, Neuropilin-1 is expressed by endothelial and tumor cells as an isoform-specific receptor for VEGF. Cell 92, 735-745 (1998).

7. T. Kawasaki, T. Kitsukawa, Y. Bekku, Y. Matsuda, M. Sanbo, T. Yagi, H. Fuisawa, A requirement for neuropilin-1 in embryonic vessel formation. Development 126, 4895-4902 (1999).

8. T. Kitsukawa, A. Shimono, A. Kawakami, H. Kondoh, H. Fujisawa, Overexpression of a membrane protein, neuropilin, in chimeric mice causes anomalies in the cardiovascular system, nervous system and limbs. Development 121, 4309-4318 (1995).

9. H. Chen, Z. He, A. Bagri, M. Tessier-Lavigne, Semaphorin-neuropilin interactions underlying sympathetic axon responses to class III semaphorins. Neuron 21, 1283-1290 (1998).

10. F. Nakamura, M. Tanaka, T. Takahashi, R. G. Kalb, S. M. Strittmatter, Neuropilin-1 extracellular domains mediate semaphorin D/III-induced growth cone collapse. Neuron 21, 1093-1100 (1998).

11. L. Roth, C. Nasarre, S. Dirrig-Grosch, D. Aunis, G. Crémel, P. Hubert, D. Bagnard, Transmembrane domain interactions control biological functions of neuropilin-1. Mol. Biol. Cell. 19, 646-654 (2008).

12. H. Cai, R. R. Reed, Cloning and characterization of neuropilin-1-interacting protein: a PSD95/Dlg/ZO-1 domain-containing protein that interacts with the cytoplasmic domain of neuropilin-1. J. Neurosci. 19, 6519-6527 (1999).

13. A. Lanahan X. Zhang, A. Fantin, Z. Zhuang, F. Rivera-Molina, K. Speichinger, C. Prahst, J. Zhang, Y. Wang, G. Davis, D. Toomre, C. Ruhrberg, M. Simons, The Neuropilin 1 Cytoplasmic Domain Is Required for VEGF-A-Dependent Arteriogenesis. Dev. Cell. 25, 156-168 (2013). 
14. S. Soker, H.-Q. Miao, M. Nomi, S. Takashima, M. Klagsbrun, VEGF165 mediates formation of complexes containing VEGFR-2 and neuropilin-1 that enhance VEGF165-receptor binding. J. Cell. Biochem. 85, 357-368 (2002).

15. C. Prahst, M. Héroult, A. A. Lanahan, N. Uziel, O. Kessler, N. Shraga-Heled, M. Simons, G. Neufeld, H. G. Augustin, Neuropilin-1-VEGFR-2 complexing requires the PDZ-binding domain of neuropilin-1. J. Biol. Chem. 283, 25110-25114 (2008).

16. D. R. Senger, S. J. Galli, A. M. Dvorak, A. Perruzi, V.S. Harvey, F. F. Dvorak, Tumor cells secrete a vascular permeability factor that promotes accumulation of ascites fluid. Science 219, 983-985 (1983).

17. B. P. Eliceiri, R. Paul, P. L. Schwartzberg, J. D. Hood, J. Leng, D. A. Cheresh, Selective requirement for Src kinases during VEGF-induced angiogenesis and vascular permeability. Mol. Cell. 4, 915-924 (1999).

18. M. T. Chou, J. Wang, D. J. Fujita, Src kinase becomes preferentially associated with the VEGFR, KDR/FIk-1, following VEGF stimulation of vascular endothelial cells. BMC Biochem. 3, 3-32 (2002).

19. Y. Wallez, F. Cand, F. Cruzalegui, C. Wernstedt, S. Souchelnytskyi, I Vilgrain, P. Huber, Src kinase phosphorylates vascular endothelial-cadherin in response to vascular endothelial growth factor: identification of tyrosine 685 as the unique target site. Oncogene 26, 1067-1077 (2006).

20. X. L. Chen, J. O. Nam, C. Jean, C. Lawson, C. T. Walsh, E. Goka, S. T. Lim, A. Tomar, I. Tancioni, S. Uryu, J. L. Guan, L. M. Acevedo, S. M. Weis, D. A. Cheresh, D. D. Schlaepfer, VEGF-induced vascular permeability is mediated by FAK. Dev. Cell. 22, 146-157 (2012).

21. Z. Sun, X. Li, S. Massena, S. Kutschera, N. Padhan, L. Gualandi, V. Sundvold-Gjerstad, K. Gustafsson, W. W. Choy, G. Zang, M. Quach, L. Jansson, M. Phillipson, M. R. Abid, A. Spurkland, L. ClaessonWelsh, VEGFR2 induces c-Src signaling and vascular permeability in vivo via the adaptor protein TSAd. J. Exp. Med. 209, 1363-1377 (2012).

22. C. Jean, X. L. Chen, J. O. Nam, I. Tancioni, S. Uryu, C. Lawson, K. K. Ward, C. T. Walsh, N. L. Miller, M. Ghassemian, P. Turowski, E. Dejana, S. Weis, D. A. Cheresh, D. D. Schlaepfer, Inhibition of endothelial FAK activity prevents tumor metastasis by enhancing barrier function. J. Cell Biol. 204, 247-263 (2014).

23. N. Lambeng, Y. Wallez, C. Rampon, F. Cand, G. Christé, D. Gulino-Debrac, I. Vilgrain, P. Huber, Vascular endothelial-cadherin tyrosine phosphorylation in angiogenic and quiescent adult tissues. Circ. Res. 96, 384-391 (2005).

24. J. Gavard, J. S. Gutkind, VEGF controls endothelial-cell permeability by promoting the $\beta$-arrestindependent endocytosis of VE-cadherin. Nat. Cell Biol. 8, 1223-1234 (2006).

25. D. Feng, J. A. Nagy, J. Hipp, H. F. Dvorak, A. M. Dvorak, Vesiculo-vacuolar organelles and the 
regulation of venule permeability to macromolecules by vascular permeability factor, histamine, and serotonin. J. Exp. Med. 183, 1981-1986 (1996).

26. S. A. Stacker, A. Vitali, C. Caesar, T. Domagala, L. C. Groenen, E. Nice, M. G. Achen, A. F. Wilks, A mutant form of vascular endothelial growth factor (VEGF) that lacks VEGF receptor-2 activation retains the ability to induce vascular permeability. J. Biol. Chem. 274, 34884-34892 (1999).

27. P. M. Becker, J. Waltenberger, R. Yachechko, T. Mirzapoiazova, J. S. Sham, C. G. Lee, J. A. Elias, A. D. Verin, Neuropilin-1 regulates vascular endothelial growth factor-mediated endothelial permeability. Circ. Res. 96, 1257-1265 (2005).

28. R. Mamluk, M. Klagsbrun, M. Detmar, D. R. Bielenberg, Soluble neuropilin targeted to the skin inhibits vascular permeability. Angiogenesis 8, 217-227 (2005).

29. T. Teesalu, K. N. Sugahara, V. R. Kotamraju, E. Ruoslahti, C-end rule peptides mediate neuropilin-1dependent cell, vascular, and tissue penetration. Proc. Natl. Acad. Sci. USA 106, 16157-16162 (2009).

30. K. N. Sugahara, T. Teesalu, P. P. Karmali, V. R. Kotamraju, L. Agemy, D. R. Greenwald, E. Ruoslahti, Coadministration of a tumor-penetrating peptide enhances the efficacy of cancer drugs. Science 328, 1031-1035 (2010).

31. H. B. Pang, G. B. Braun, T. Friman, P. Aza-Blanc, M. E. Ruidiaz, K. N. Sugahara, T. Teesalu, E. Ruoslahti, An endocytosis pathway initiated through neuropilin-1 and regulated by nutrient availability. Nat. Commun. 5, 4904 (2014).

32. H. Gitay-Goren, T. Cohen, S. Tessler, S. Soker, S. Gengrinovitch, P. Rockwell, M. Klagsbrun, B. Z. Levi, G. Neufeld, Selective binding of VEGF121 to one of the three vascular endothelial growth factor receptors of vascular endothelial cells. J. Biol. Chem. 271, 5519-5523 (1996).

33. M. W. Parker, P. Xu, X. Li, C. W. Vander Kooi, Structural basis for selective vascular endothelial growth factor-A (VEGF-A) binding to neuropilin-1. J. Biol. Chem. 287, 11082-11089 (2012).

34. N. Haspel, D. Zanuy, R. Nussinov, T. Teesalu, E. Ruoslahti, C. Aleman, Binding of a C-end rule peptide to the neuropilin-1 receptor: a molecular modeling approach. Biochemistry 50, 1755-1762 (2011).

35. M. J. Renzi, L. Feiner, A. M. Koppel, J. A. Raper, A dominant negative receptor for specific secreted semaphorins is generated by deleting an extracellular domain from neuropilin-1. J. Neurosci. 19, 7870-7880 (1999).

36. A. Antipenko, J. P. Himanen, K. van Leyen, V. Nardi-Dei, J. Lesniak, W. A. Barton, K. R. Rajashankar, M. Lu, C. Hoemme, Structure of the semaphorin-3A receptor binding module. Neuron 39, 589-598 (2003).

37. C. W. Vander Kooi, M. A. Jusino, B. Perman, D. B. Neau, H. D. Bellamy, D. J. Leahy, Structural basis for ligand and heparin binding to neuropilin B domains. Proc. Natl. Acad. Sci. U.S.A. 104, 6152-6157 
(2007).

38. Z. Gluzman-Poltorak, T. Cohen, Y. Herzog, G. Neufeld, Neuropilin-2 and neuropilin-1 are receptors for the 165-amino acid form of vascular endothelial growth factor (VEGF) and of placenta growth factor-2, but only neuropilin-2 functions as a receptor for the 145-amino acid form of VEGF. J. Biol. Chem. 275, 18040-18045 (2000).

39. L. Roth, L. Agemy., V. R. Kotamraju, G. Braun, T. Teesalu, K. N. Sugahara, J. Hamzah, E. Ruoslahti, Transtumoral targeting enabled by a novel neuropilin-binding peptide. Oncogene 31, 3754-3763 (2011)

40. N. Bayer, D. Schober, E. Prchla, R. F. Murphy, D. Blaas, R. Fuchs, Effect of bafilomycin A1 and nocodazole on endocytic transport in HeLa cells: implications for viral uncoating and infection. J. Virol. 72, 9645-9655 (1998).

41. J. Thyberg, S. Moskalewski, Role of microtubules in the organization of the Golgi complex. Exp. Cell Res. 246, 263-279 (1999).

42. L. Lu, R. N. Hannoush, B. C. Goess, S. Varadarajan, M. D. Shair, T. Kirchhausen, The small molecule dispergo tubulates the endoplasmic reticulum and inhibits export. Mol. Biol. Cell. 24, 1020-1029 (2013).

43. P. D. Collins, D. T. Connolly, T. J. Williams, Characterization of the increase in vascular permeability induced by vascular permeability factor in vivo. Br. J. Pharmacol. 109, 195-199 (1993).

44. P. M. Becker, A. D. Verin, M. A. Booth, F. Liu, A. Birukava, J. G. Garcia, Differential regulation of diverse physiological responses to VEGF in pulmonary endothelial cells. Am. J. Physiol. Lung Cell Mol. Physiol. 281, L1500-11 (2001).

45. E. Ackah, J. Yu, S. Zoellner, Y. Iwakiri, C. Skurk, R. Shibata, N. Ouchi, R. M. Easton, G. Galasso, M. J. Birnbaum, K. Walsh, W. C. Sessa, Akt1/protein kinase B is critical for ischemic and VEGF-mediated angiogenesis. J. Clin. Invest. 115, 2119-2127 (2005).

46. L. M. Acevedo, S. Barillas, S. M. Weis, J. R. Gothert, D. A. Cheresh, Semaphorin 3A suppresses VEGFmediated angiogenesis yet acts as a vascular permeability factor. Blood 111, 2674-2680 (2008).

47. J. M. Wood, G. Bold, E. Buchdunger, R. Cozens, S. Ferrari, J. Frei, F. Hofmann, J. Mestan, H. Mett, T. O'Reilly, E. Persohn, J. Rösel, C. Schnell, D. Stover, A. Theuer, H. Towbin, F. Wenger, K. Woods-Cook, A. Menrad, G. Siemeister, M. Schirner, K. H. Thierauch, M. R. Schneider, J. Drevs, G. Martiny-Baron, F. Totzke, PTK787/ZK 222584, a novel and potent inhibitor of vascular endothelial growth factor receptor tyrosine kinases, impairs vascular endothelial growth factor-induced responses and tumor growth after oral administration. Cancer Res. 60, 2178-2189 (2000).

48. A. Fantin, Q. Schwarz, K. Davidson, E. M. Normando, L. Denti, C. Ruhrberg, The cytoplasmic domain of neuropilin 1 is dispensable for angiogenesis, but promotes the spatial separation of retinal 
arteries and veins. Development 138, 4185-4191 (2011).

49. C. Nasarre, M. Roth, L. Jacob, L. Roth, E. Koncina, A. Thien, G. Labourdette, P. Poulet, P. Hubert, G. Crémel, G. Roussel, D. Aunis, D. Bagnard, Peptide-based interference of the transmembrane domain of neuropilin-1 inhibits glioma growth in vivo. Oncogene 29, 2381-2392 (2010).

50. H. Q. Miao, S. Soker, L. Feiner, J. L. Alonso, J. A. Raper, M. Klagsbrun, Neuropilin-1 mediates collapsin-1/semaphorin III inhibition of endothelial cell motility: functional competition of collapsin1 and vascular endothelial growth factor-165. J. Cell Biol. 146, 233-242 (1999).

51. C. Gu, B. J. Limberg, G. B. Whitaker, B. Perman, D. J. Leahy, J. S. Rosenbaum, D. D. Ginty, A. L. Kolodkin, Characterization of neuropilin-1 structural features that confer binding to semaphorin $3 \mathrm{~A}$ and vascular endothelial growth factor 165. J. Biol. Chem. 277, 18069-18076 (2002).

52. B. A. Appleton, P. Wu, J. Maloney, J. Yin, W.C. Liang, S. Stawicki, K. Mortara, K. K. Bowman, J. M. Elliott, W. Desmarais, J. F. Bazan, A. Bagri, M. Tessier-Lavigne, A. W. Koch, Y. Wu, R. J. Watts, C. Wiesman, Structural studies of neuropilin/antibody complexes provide insights into semaphorin and VEGF binding. EMBO J. 26, 4902-4912 (2007).

53. M. W. Parker, L. M. Hellman, P. Xu, M. G. Fried, C. W. Vander Kooi, Furin processing of semaphorin $3 \mathrm{~F}$ determines its anti-angiogenic activity by regulating direct binding and competition for neuropilin. Biochemistry 49, 4068-4075 (2010).

54. J. A. Nagy, L. Benjamin, H. Zeng, A. M. Dvorak, H. F. Dvorak, Vascular permeability, vascular hyperpermeability and angiogenesis. Angiogenesis 11, 109-119 (2008).

55. L. Yuan, D. Moyon, L. Pardanaud, C. Bréant, M. J. Karkkainen, K. Alitalo, A. Eichmann, Abnormal lymphatic vessel development in neuropilin 2 mutant mice. Development 129, 4797-4806 (2002).

56. V. Joukov, T. Sorsa, V. Kumar, M. Jeltsch, L. Claesson-Welsh, Y. Cao, O Saksela, N. Kalkkinen, K. Alitalo, Proteolytic processing regulates receptor specificity and activity of VEGF-C. EMBO J. 16, 3898-3911 (1997).

57. M. Jeltsch, A. Kaipainen, V. Joukov, X. Meng, M. Lakso, H. Rauvala, M. Swartz, D. Fukumura, R. K. Jain, K. Alitalo, Hyperplasia of lymphatic vessels in VEGF-C transgenic mice. Science 276, 1423-1425 (1997).

58. L. Wang, H. Zeng, P. Wang, S. Soker, D. Mukhopadhyay, Neuropilin-1-mediated vascular permeability factor/vascular endothelial growth factor-dependent endothelial cell migration. J. Biol. Chem. 278, 48848-48860 (2003).

59. L. Wang, S. K. Dutta, T. Kojima, X. Xu, R. Khosravi-Far, S. C. Ekker, D. Mukhopadhyay, Neuropilin-1 modulates p53/caspases axis to promote endothelial cell survival. PLOS ONE 2, e1161 (2007). 
Acknowledgments: We thank Dr. Tim Holland-Letz for his guidance in statistical analyses and Eva Besemfelder (DKFZ Heidelberg) for excellent technical support. We would also like to thank the DKFZ Laboratory Animal Facility and the DKFZ Light Microscopy Core Facilities. Funding: This work was supported by grants from the SFB-TR23 "Vascular Differentiation and Remodeling" [project A3, to H.G.A.]), the SFB873 "Maintenance and Differentiation of Stem Cells in Development and Disease" [project B6, to H.G.A.]), and the Leducq Transatlantic Network of Excellence "Lymph Vessels in Obesity and Cardiovascular Disease" [to H.G.A.], and by a Welcome Trust Junior Investigator Award (ref. 095623/Z/11/Z) [to C.R.]. H.G.A. is supported by an endowed chair from the Aventis Foundation. Author contributions: L.R. designed and performed research, analyzed data and wrote the article; C.P. designed research and performed experiments; T.R., S.S., S.W., M.H. and M.R. performed experiments; A.F. and C.R. designed research and provided the NRP1cyto ${ }^{--}$mice; H.G.A. designed research, analyzed data and wrote the article. Competing interests: The authors declare that they have no competing interests. 


\section{Roth et al., Fig. 1}
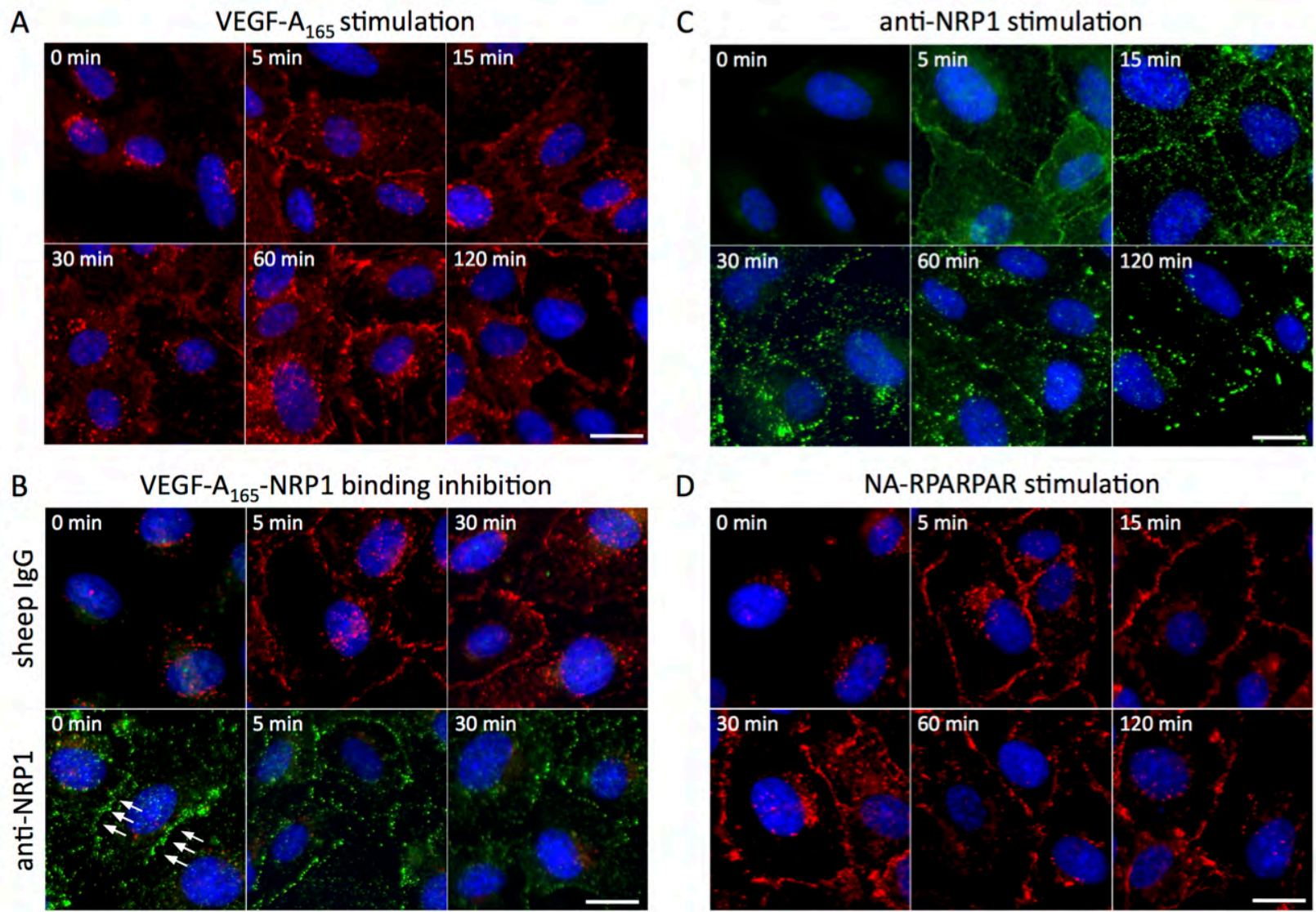

$\mathrm{D}$

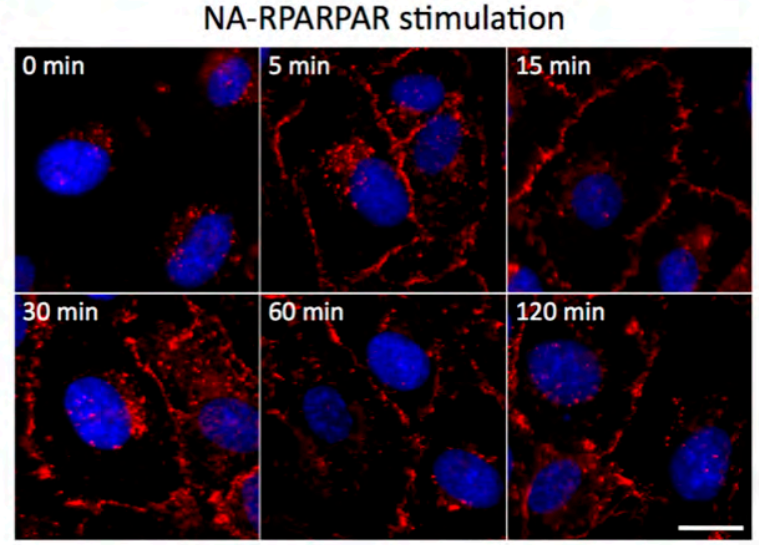

Fig. 1. VEGF-A $A_{165}$, a ligand-blocking anti-NRP1 and a tetrameric CendR peptide induce NRP1 accumulation at endothelial cell-cell contacts. (A-D) Epifluorescence microscope images of permeabilized HUVEC monolayers. (A) Cells were stimulated with VEGF-A 165 and stained with an antibody against NRP1 (red). (B) After incubation with the ligand blocking anti-NRP1 antibody (antiNRP1) (lower panel) or its corresponding control sheep IgG (upper panel), cells were stimulated with VEGF-A 165 . HUVECs were stained with an antibody specific for NRP1 (red) and secondary anti-sheep antibody (green). Accumulation of NRP1 at cell-cell contacts was observed in absence of VEGF-A 165 (white arrows). (C) Cells were stimulated with anti-NRP1 and stained with secondary anti-sheep antibody (green). (D) Cells were stimulated with NA-RPARPAR peptide and stained with an antibody specific for NRP1 (red). Nuclei were stained with Hoechst (blue). Images in (A) to (D) are representative of N>3 independent experiments. Scale bar: $20 \mu \mathrm{m}$. 


\section{Roth et al., Fig. 2}

A

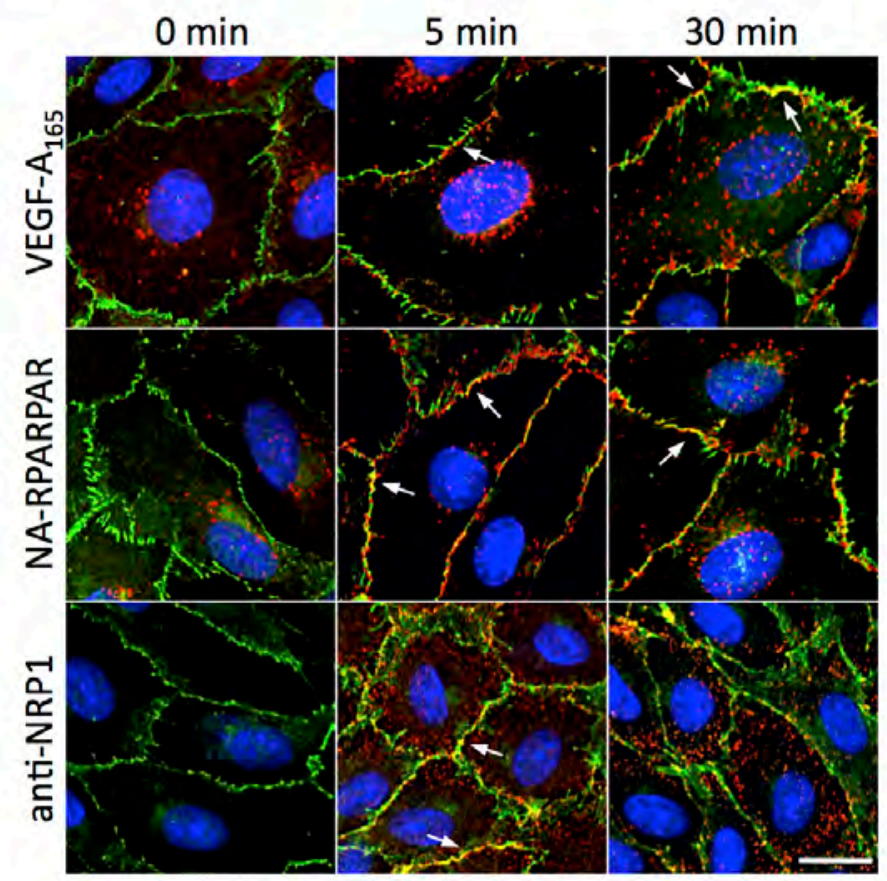

B In vitro endothelial leakage

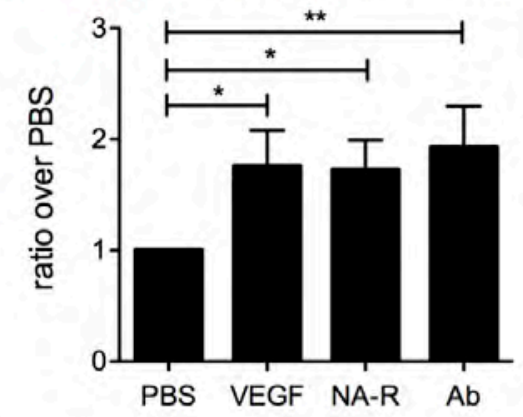

C In vivo vascular leakage

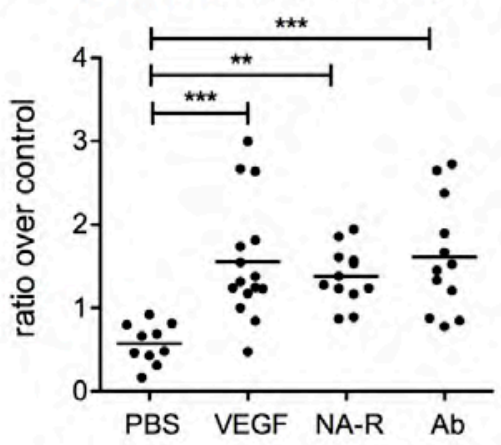

Fig. 2. VEGF-A $A_{165}, N A-R P A R P A R$ and anti-NRP1 induce NRP1 junctional localization and endothelial leakage in vitro and in vivo. (A) Confocal microscopy images of permeabilized HUVEC monolayers stimulated with VEGF-A $A_{165}$, NA-RPARPAR and anti-NRP1, and stained with an antibody against VEcadherin (green). NRP1 (red) was stained with an antibody against NRP1 (upper and middle panels) or directly with the secondary anti-sheep antibody (lower panel). Nuclei were stained with Hoechst (blue). White arrows indicate co-localization between NRP1 and VE-cadherin. Images are representative of N=4 independent experiments. Scale bar: $20 \mu \mathrm{m}$. (B) HUVEC monolayers were seeded on top of Transwell filters, and stimulated with PBS, VEGF-A 165 (VEGF), NA-RPARPAR (NA-R) and anti-NRP1 (Ab). Leakage of FITC-dextran $70 \mathrm{kDa}$ from the upper to the lower well was measured by comparing fluorescence values at $520 \mathrm{~nm}$. (mean $\pm \mathrm{SEM} ; \mathrm{N}=9$ independent experiments; the median from $\mathrm{n}=3-6$ replicates per independent experiment was used for statistical analyses; Friedman test followed by Dunns' multiple comparison post hoc test; ${ }^{*}, \mathrm{p}<0.05 ;{ }^{* *}, \mathrm{p}<0.01$ ). (C) Wild-type mice were systemically injected with Evans Blue, followed by injection of PBS, VEGF-A ${ }_{165}$, NA-RPARPAR, anti-NRP1 antibody (Ab), and of their respective controls. The extravasated dye concentration was measured at $620 \mathrm{~nm}$ and results expressed as a ratio between the tested substance and its control (mean; $\mathrm{N}=10-15$ mice per treatment; KruskalWallis test followed by Dunns' multiple comparison post hoc test; ${ }^{* *}, \mathrm{p}<0.01 ;{ }^{* * *}, \mathrm{p}<0.001$, ). 


\section{Roth et al., Fig. 3}

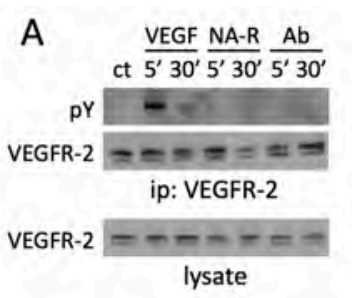

C

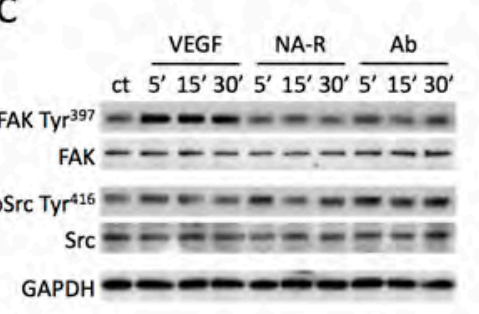

B
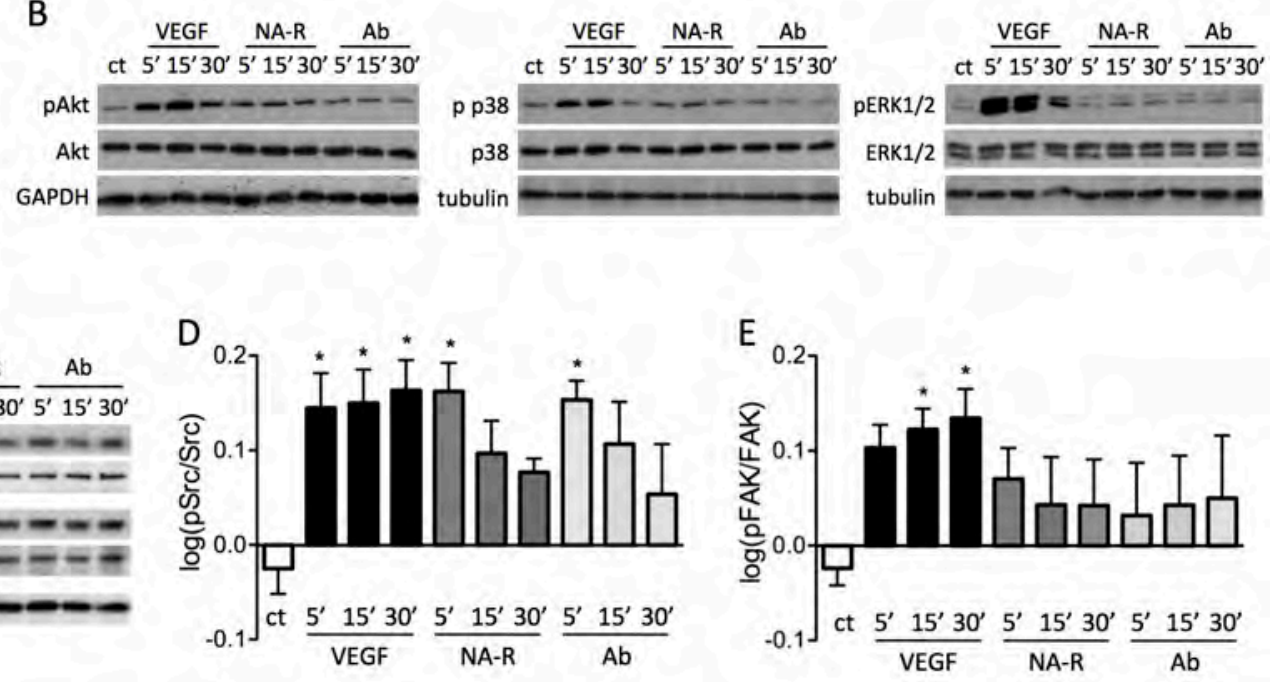

Fig. 3. Unlike VEGF-A 165, NA-RPARPAR and anti-NRP1 do not activate VEGFR-2, Akt, p38, ERK and FAK. (A-C) HUVEC monolayers were stimulated with VEGF-A 165 (VEGF), NA-RPARPAR (NA-R), or anti-NRP1 antibody (Ab). (A) Cell lysates were immunoprecipitated with an antibody against VEGFR-2, and blotted with anti-VEGFR-2 and anti-phosphotyrosine antibodies. The corresponding total lysates were blotted for VEGFR-2. N=4 independent experiments. (B, C) Blotting was performed on total lysates with antibodies against pAkt, pERK1/2, p-p38 ( $N=3$ independent experiments) (B), pSrc Y416, pFAK Y397 ( $N=5$ (SrC) and 7 (FAK) independent experiments) (C), against the corresponding total proteins, and against the loading controls GAPDH and tubulin. (D, E) Intensities resulting from pSrc and Src blottings (D) and pFAK and FAK blottings (E) were quantified (mean \pm SEM; $N=5$ (Src) and 7 (FAK) independent experiments; paired ANOVA test followed by Tukey's multiple comparison post hoc test; ${ }^{*}, \mathrm{p}<0.05 ;{ }^{* *}$, $\mathrm{p}<0.01)$. 
Roth et al., Fig. 4 
A

B
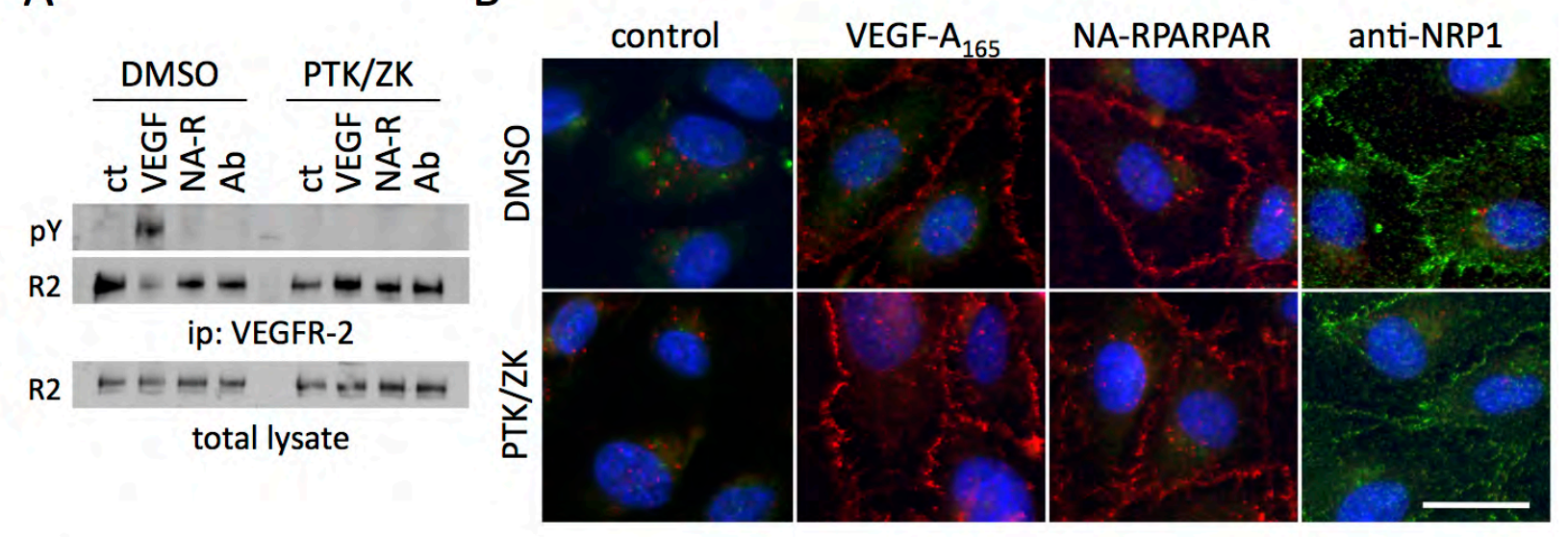

C

D
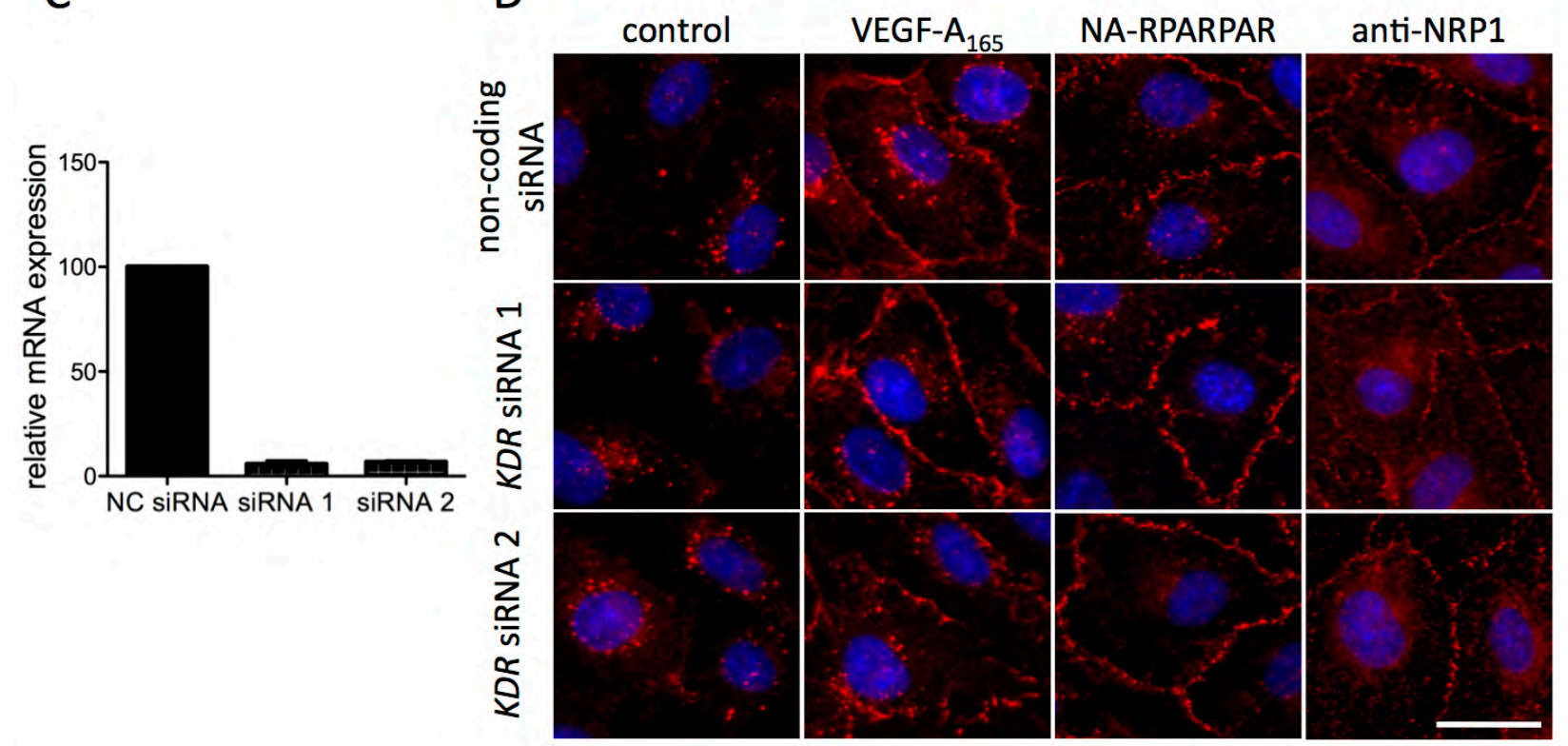

E

F

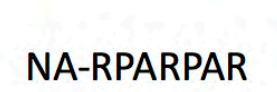

G
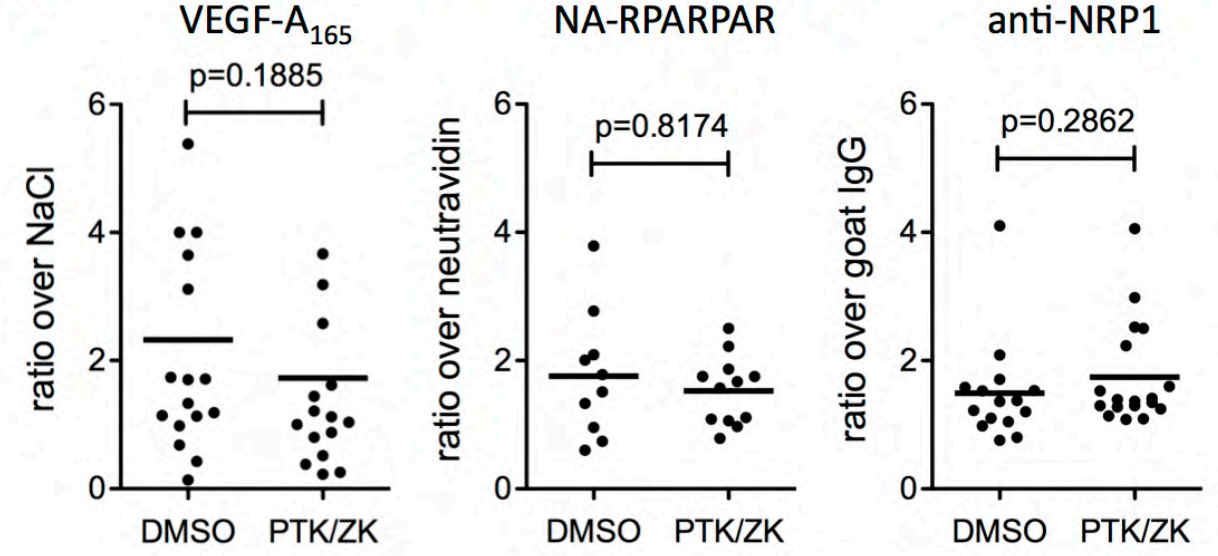

H

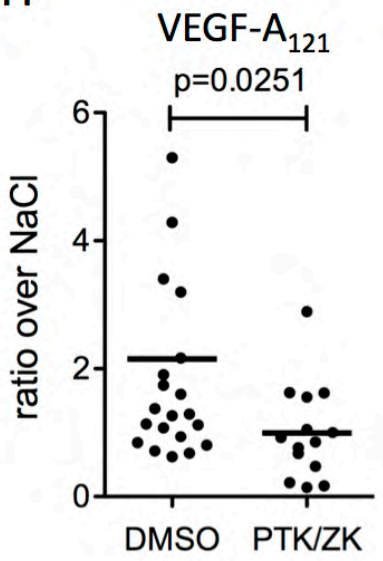


Fig. 4. VEGF-A $A_{165}, N A-R P A R P A R$ and anti-NRP1 induce NRP1 relocalization and vascular leakage independently of VEGFR-2. (A, B) HUVEC monolayers were treated with PTK/ZK or DMSO before stimulation. (A) Cell lysates were immunoprecipitated with VEGFR-2 antibody. Immunoprecipitates were blotted for VEGFR-2 and phosphotyrosine, and corresponding total lysates for VEGFR-2 (R2). Representative scans of $\mathrm{N}=5$ experiments. (B) HUVEC monolayers were stained for NRP1 (red), nuclei were stained with Hoechst (blue), and cells were imaged with an epifluorescence microscope. Representative images from $\mathrm{N}=3$ experiments. Scale bar: $25 \mu \mathrm{m}$. (C, D) HUVECs were transfected with non-coding (NC) siRNA, or siRNAs coding for VEGFR-2 (KDR) (siRNA1 and siRNA2). (C) KDR mRNA relative expression was quantified by qRT-PCR (mean $\pm \mathrm{SEM} ; \mathrm{N}=3$ independent experiments). (D) Epifluorescence images of transfected HUVECs in (C), stimulated with VEGF-A $A_{165}$ (VEGF), NA-RPARPAR (NA-R), or anti-NRP1 (Ab). Cells were stained for NRP1 (red) and nuclei were stained with Hoechst (blue). Representative images of $\mathrm{N}=3$ experiments. Scale bar: $20 \mu \mathrm{m}$. (E-H) Mice were injected intraperitoneally with DMSO or PTK/ZK before systemic treatment with Evans Blue. Leakage was induced with VEGF-A $\mathrm{A}_{165}$ (E), NA-RPARPAR (F), anti-NRP1 (G), or VEGF-A $121(\mathbf{H})$, and results expressed as a ratio between the tested substance and its respective control (mean; $\mathrm{N}=10-20$ mice per condition; Mann-Whitney test). 


\section{Roth et al., Fig. 5}

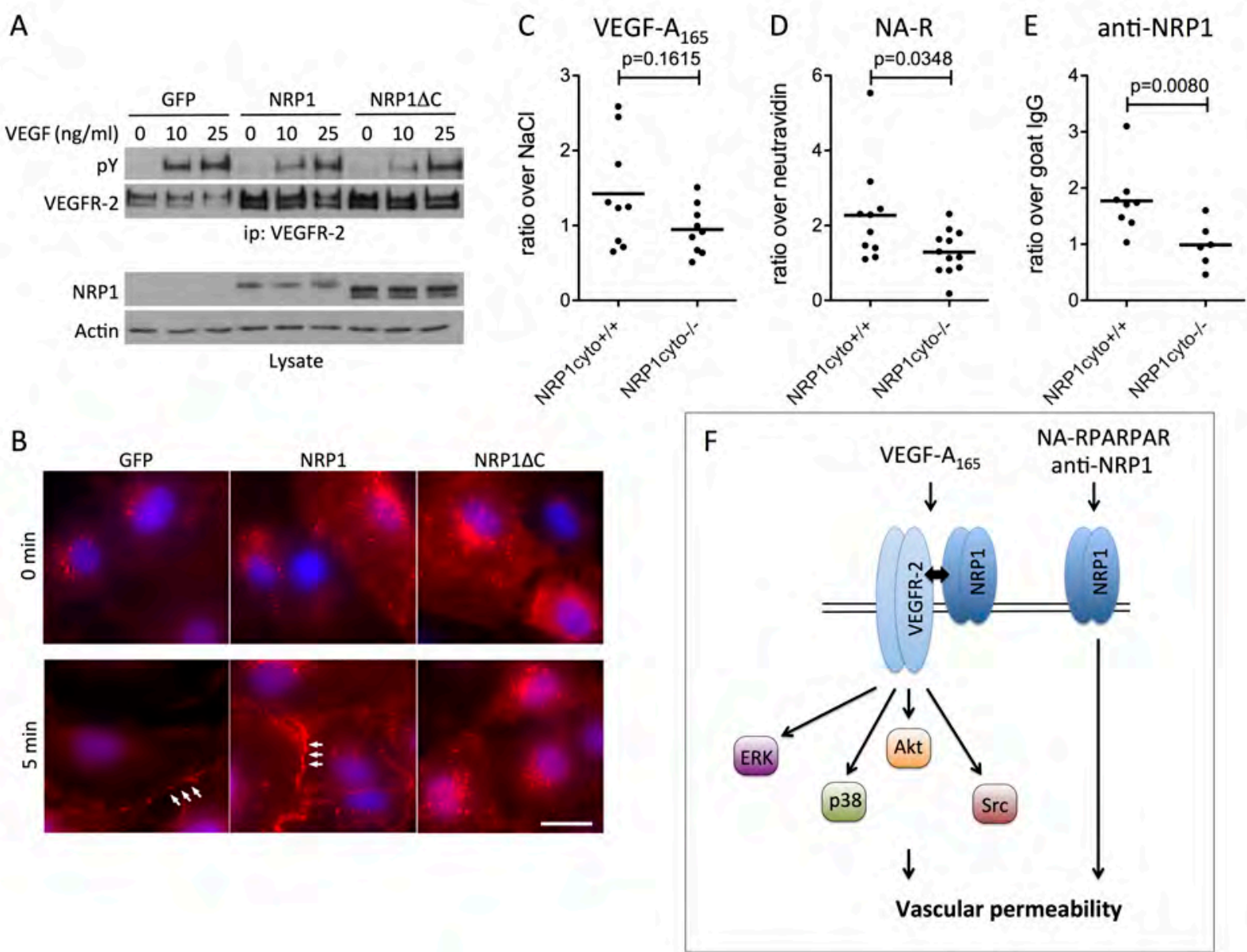

Fig. 5. The NRP1 cytoplasmic domain mediates vascular leakage. (A, B) HUVECs overexpressing GFP, fulllength NRP1 or NRP1 deprived from the cytoplasmic domain (NRP1 $\triangle C$ ) were stimulated with VEGF- $A_{165}$. (A) Cell lysates were immunoprecipitated with VEGFR-2 antibody. Immunoprecipitates were blotted for VEGFR-2 and phosphotyrosine, and total lysates for NRP1 and actin. N=3 independent experiments. (B) HUVECs overexpressing GFP, NRP1 or NRP1 $\triangle C$ were stained with anti-NRP1 (red). Nuclei were stained with Hoechst (blue). Representative images of $\mathrm{N}=3$ independent experiments. Scale bar: $20 \mu \mathrm{m}$. (C-E). Wild-type mice (NRP1cyto ${ }^{+/+}$) and mice expressing cytoplasmatically truncated NRP1 (NRP1cyto ${ }^{-/}$) were injected with Evans Blue. Leakage was induced with VEGF-A 165 (VEGF) (C), NA-RPARPAR (NA-R) (D), and anti-NRP1 $(A b)(E)$, and results expressed as a ratio between the tested substance and its respective control (mean; N=6-12 mice per group; Mann-Whitney test). (F) Schematic representation of NRP1induced vascular permeability. VEGF- $A_{165}$ induces VEGFR-2-mediated permeability through phosphorylation of ERK1/2, p38, Akt and Src, likely in conjunction with NRP1. The non-VEGFR-2 binding ligands NA-RPARPAR and anti-NRP1 induce leakage through the NRP1 cytoplasmic domain independently of VEGFR-2. 


\section{Supplementary Materials}

\section{Neuropilin-1 mediates vascular permeability independently of vascular endothelial growth factor receptor-2 activation}

Lise Roth, Claudia Prahst, Tina Ruckdeschel, Soniya Savant, Simone Weström, Alessandro Fantin, Maria Riedel, Mélanie Héroult, Christiana Ruhrberg, and Hellmut G. Augustin 
Roth et al., Suppl. Fig. S1

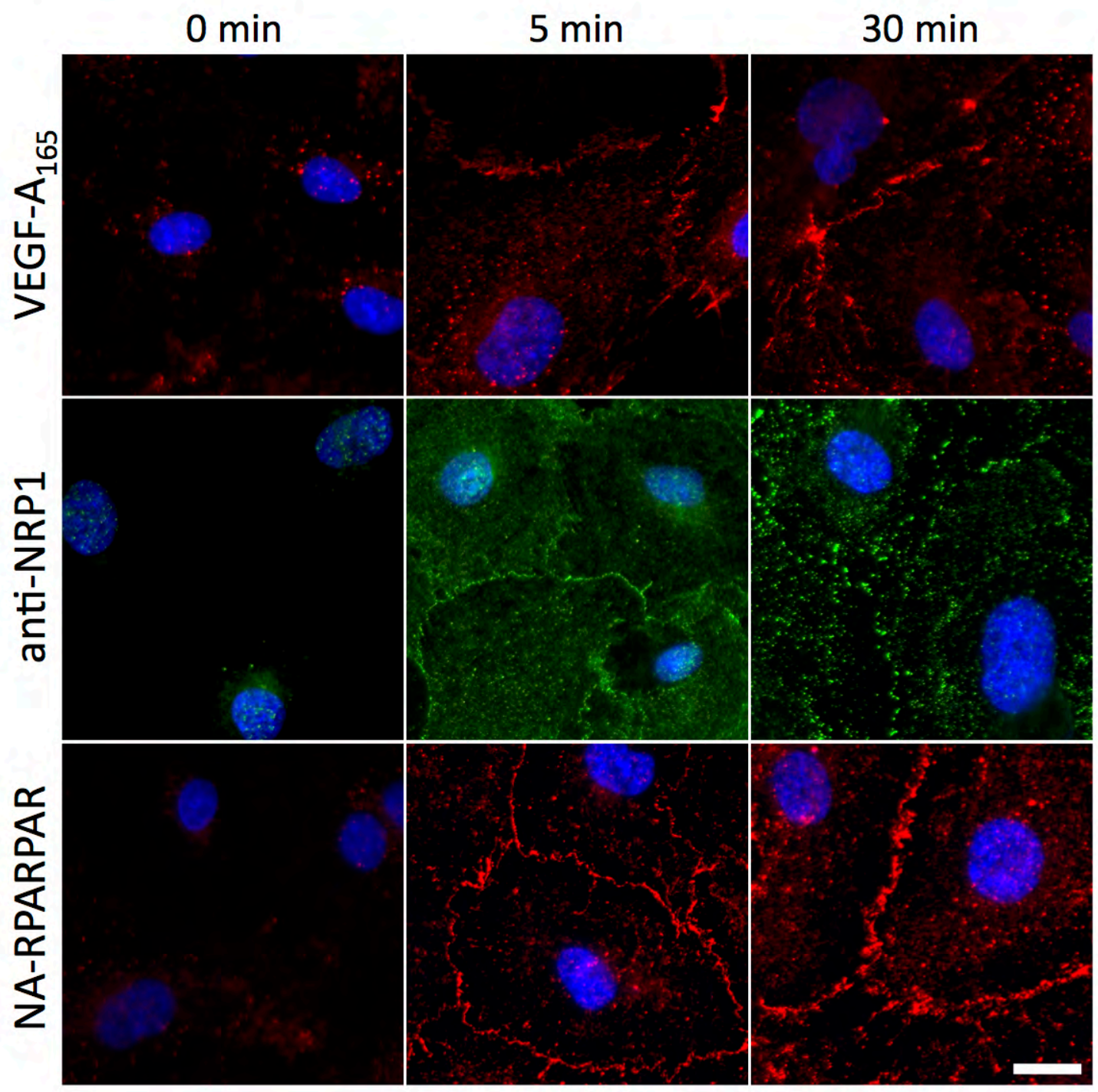

Figure S1. VEGF-A $A_{165}$, the ligand-blocking anti-NRP1 antibody and NA-RPARPAR induce NRP1 accumulation at cell-cell contacts in HDBECs. HDBEC monolayers were stimulated with VEGF- $\mathrm{A}_{165}$ (upper panel), anti-NRP1 (middle panel) or NA-RPARPAR (lower panel) prior to fixation, permeabilization and staining with an antibody against NRP1 (red) or directly with a secondary antibody anti-sheep (green). Nuclei were stained with Hoechst (blue). Cells were imaged using an epifluorescence microscope. Representative images from $\mathrm{N}>3$ experiments with similar findings are shown. Scale bar: $20 \mu \mathrm{m}$. 
Roth et al., Suppl. Fig. S2

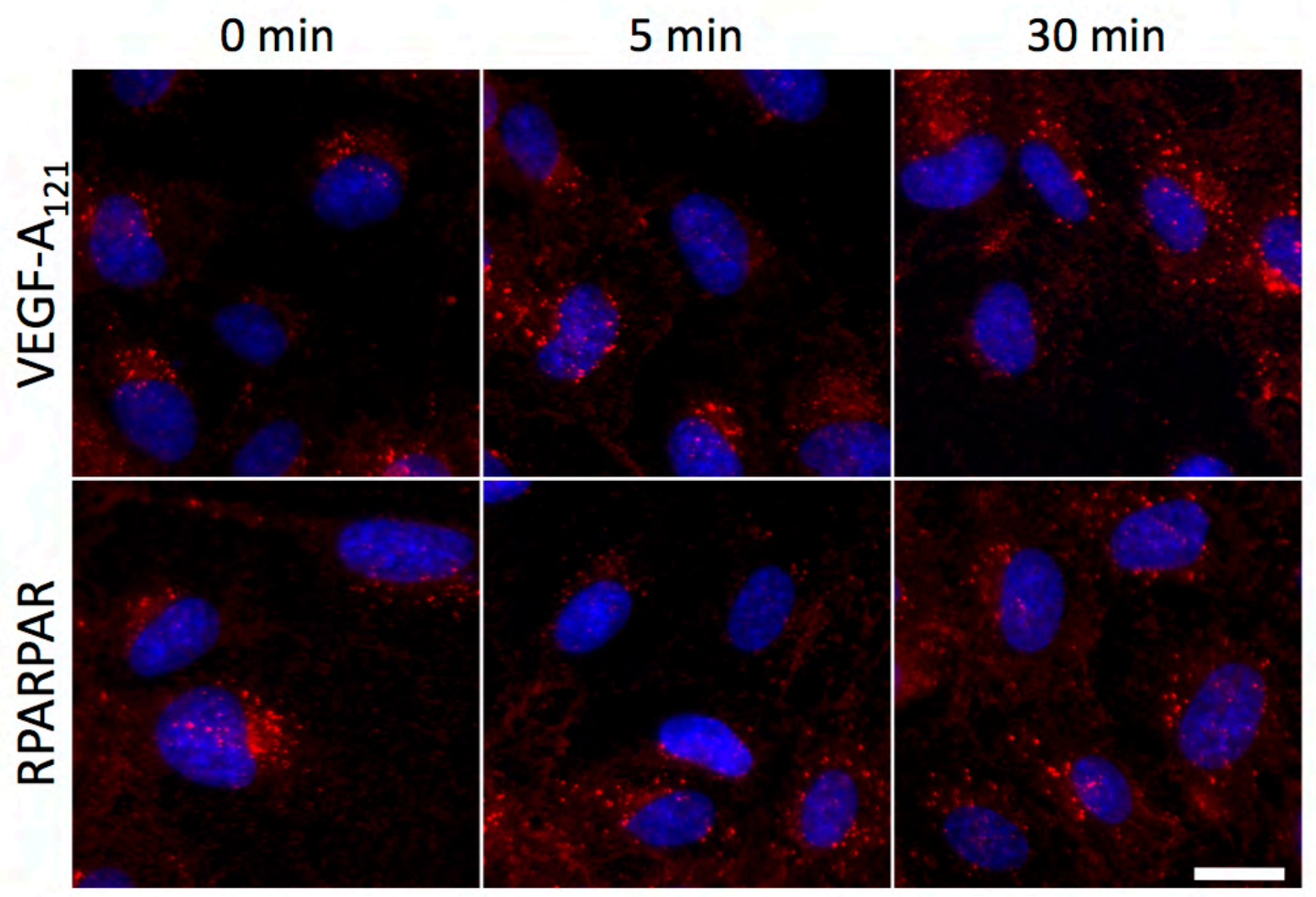

Figure S2. VEGF-A $A_{121}$ and monomeric RPARPAR do not induce NRP1 accumulation at endothelial cell-cell contacts. HUVEC monolayers were stimulated with VEGF-A $A_{121}$ or RPARPAR $(1 \mu \mathrm{M})$ for 5 and 30 min prior to fixation, permeabilization and staining with an antibody against NRP1 (red). Nuclei were stained with Hoechst (blue). Cells were imaged using an epifluorescence microscope. Representative images from $\mathrm{N}>3$ experiments are shown. Scale bar: $20 \mu \mathrm{m}$. 
Roth et al., Suppl. Fig. S3

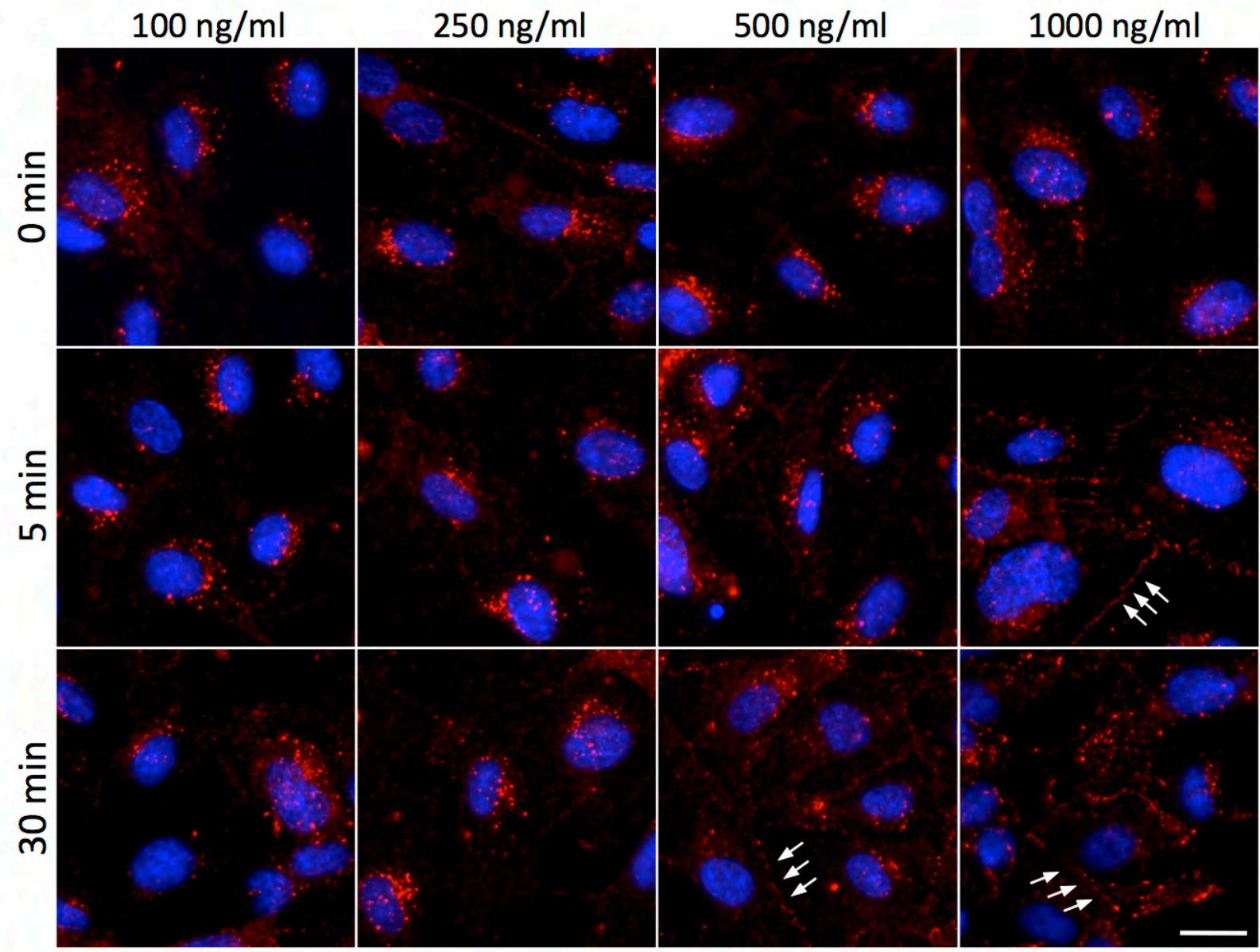

Figure S3. High concentrations of Sema3A induce weak NRP1 accumulation at cell-cell contacts. HUVEC monolayers were stimulated with Sema3A prior to fixation, permeabilization and staining with an antibody against NRP1 (red). Note the weak relocalization at cell-cell contacts induced by $500 \mathrm{ng} / \mathrm{ml}$ and $1000 \mathrm{ng} / \mathrm{ml} \mathrm{Sema3A}$ after 5 or $30 \mathrm{~min}$ (white arrows). Nuclei were stained with Hoechst (blue). Cells were imaged using an epifluorescence microscope. Representative images from $\mathrm{N}=3$ experiments are shown. Scale bar: $20 \mu \mathrm{m}$. 
Roth et al., Suppl. Fig. S4

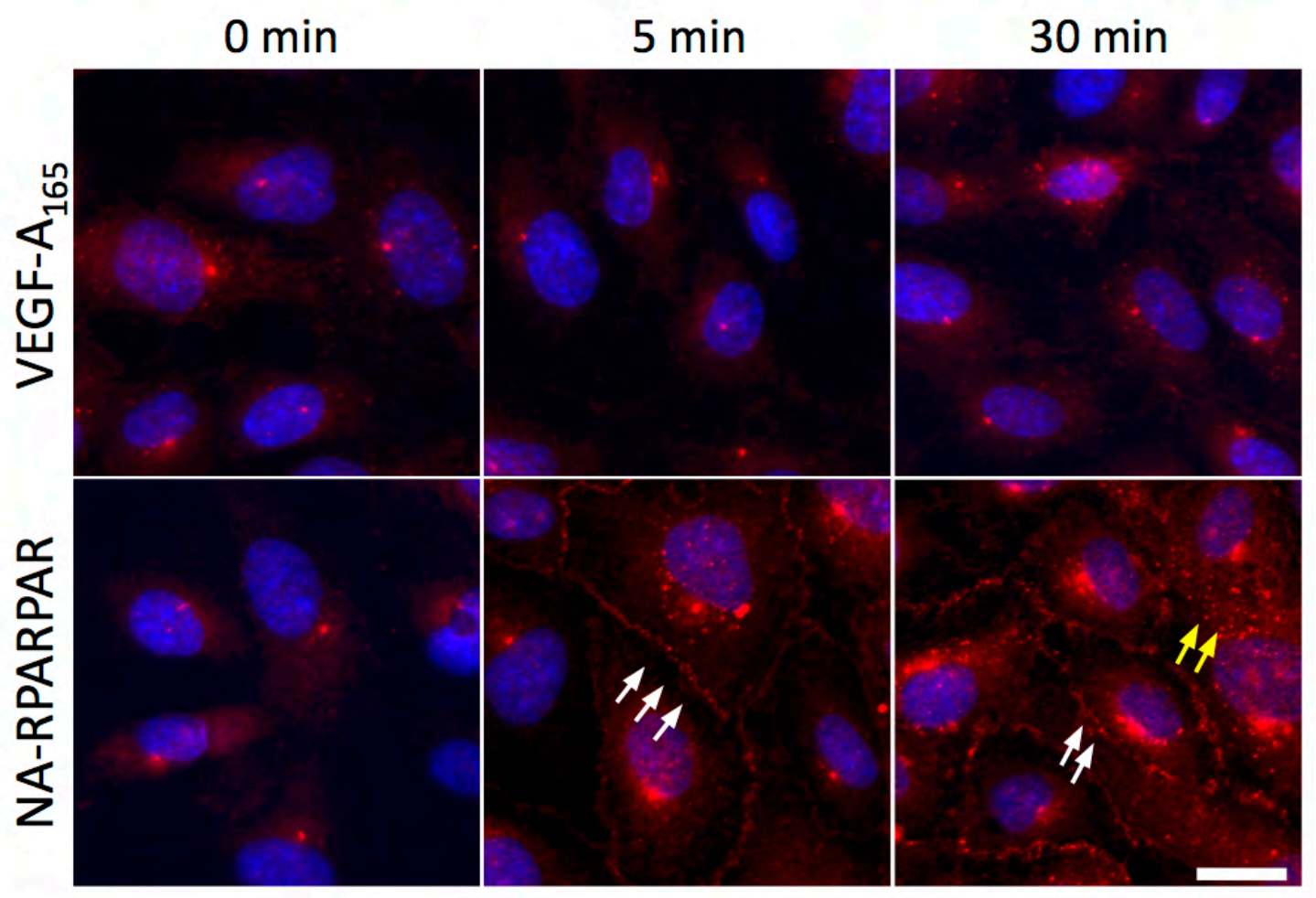

Figure S4. NRP2 accumulates at cell-cell contacts upon stimulation with NA-RPARPAR stimulation, but not with VEGF-A $A_{165}$. HUVEC monolayers were stimulated with VEGF-A ${ }_{165}$ (upper panel) or NA-RPARPAR (lower panel) prior to fixation, permeabilization and staining with an antibody against NRP2 (red). NARPARPAR induced NRP2 accumulation at cell-cell contacts (white arrows) and internalization (yellow arrows). Nuclei were stained with Hoechst (blue). Cells were imaged using an epifluorescence microscope. Representative results from $\mathrm{N}=2$ experiments are shown. Scale bar: $20 \mu \mathrm{m}$. 
Roth et al., Suppl. Fig. S5

A

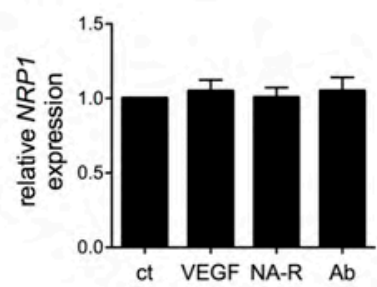

D

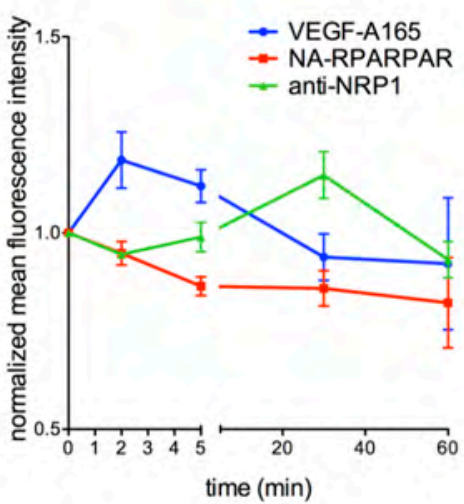

B
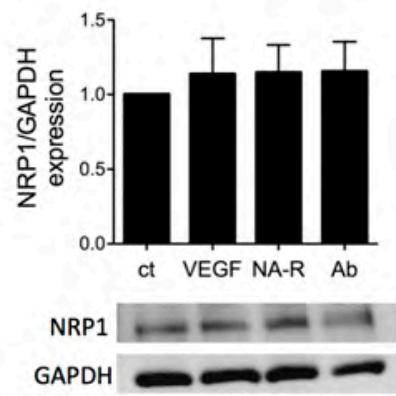

C Non permeabilized Permeabilized

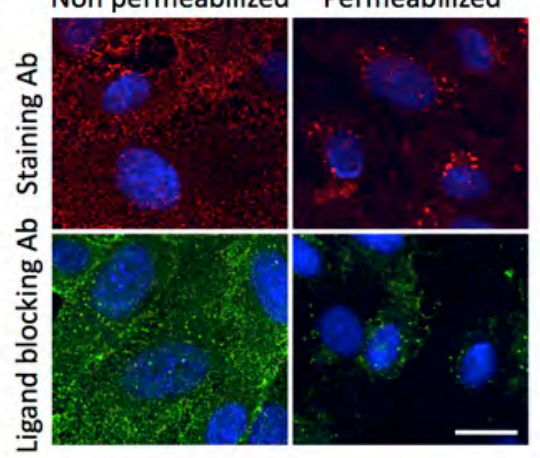

$\mathrm{E}$

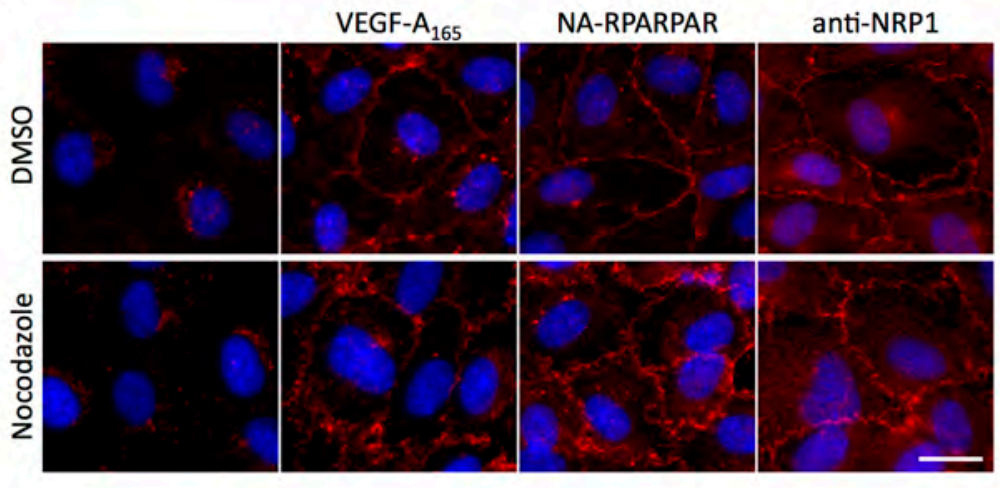

Figure S5. NRP1 relocalizes at cell-cell contacts laterally through the plasma membrane. (A, B) HUVEC monolayers were stimulated with VEGF-A ${ }_{165}$ (VEGF), NA-RPARPAR (NA-R), or anti-NRP1 antibody (Ab). (A) NRP1 mRNA relative expression was quantified by qPCR and the ratio NRP1:ACTB is shown (mean \pm SEM; $N=3$ independent experiments; Kruskal-Wallis test). (B) Blotting was performed on total lysates with antibodies against NRP1 and GAPDH. Resulting intensities were quantified and results expressed as a ratio over GAPDH (mean \pm SEM; $N=3$ independent experiments; Friedman test). (C) HUVEC monolayers were fixed with PFA 4\% (non-permeabilized) or with aceton/MeOH (permeabilized) and stained with the monoclonal mouse anti-NRP1 (staining $A b, r e d$ ) or with the ligand-blocking sheep anti-NRP1 (ligand blocking $\mathrm{Ab}$, green). Nuclei were stained with Hoechst (blue). Representative confocal images from $\mathrm{N}=2$ independent experiments. Scale bar: $20 \mu \mathrm{m}$. (D) NRP1 surface expression in HUVECs was analyzed by 
flow cytometry. Unfixed cells were stained with conjugated mouse-anti-NRP1-FITC. Results show mean fluorescence intensities normalized to the mean fluorescence intensity resulting from non-stimulated cells and analysed with FlowJo software. (mean $\pm \mathrm{SEM}$; $\mathrm{N}=3$ independent experiments). (E) After 30 min pre-incubation with nocodazole or DMSO, HUVEC monolayers were stimulated with VEGF-A $\mathrm{A}_{165}$, NARPARPAR or anti-NRP1. Cells were stained with an antibody against NRP1 or directly with a secondary antibody anti-sheep. Nuclei were stained with Hoechst (blue). Representative epifluorescence images from $\mathrm{N}=3$ independent experiments. Scale bar: $20 \mu \mathrm{m}$. 
Roth et al., Suppl. Fig. S6

A

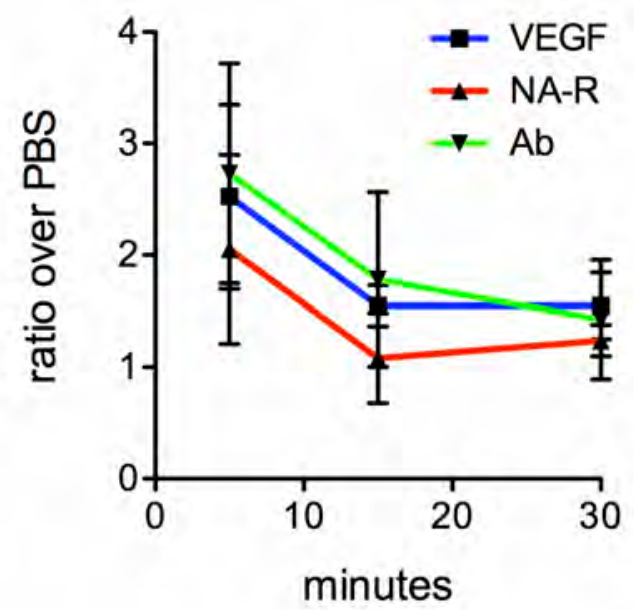

B

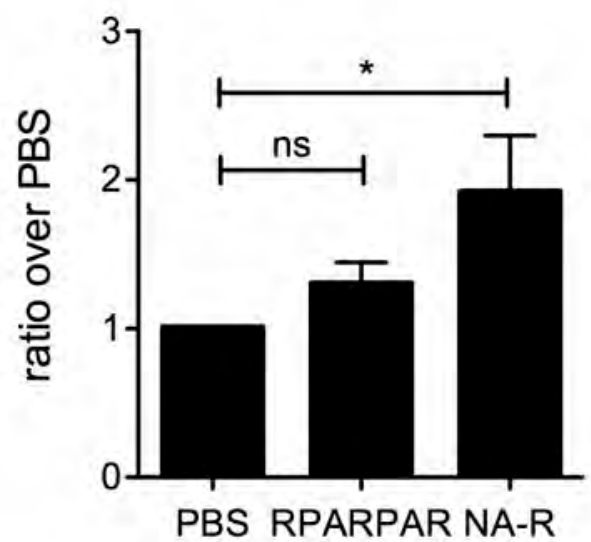

Figure S6. Maximum endothelial leakage is observed after $5 \mathrm{~min}$, and monomeric RPARPAR does not induce endothelial leakage. (A, B) HUVEC monolayers were seeded on top of Transwell filters, and leakage of FITC-dextran $70 \mathrm{kDa}$ from the upper to the lower well was measured by comparing fluorescence values at $520 \mathrm{~nm}$. (A) Cells were stimulated with PBS, VEGF-A $\mathrm{A}_{165}$ (VEGF), NA-RPARPAR (NA$\mathrm{R})$ and anti-NRP1 (Ab) for 5, 15 and $30 \mathrm{~min}$. Note that the maximum leakage was observed after $5 \mathrm{~min}$. (mean $\pm \mathrm{SEM} ; \mathrm{N}=3$ independent experiments; $\mathrm{n}=4$ replicates per experiment). (B) Cells were stimulated with PBS, monomeric RPARPAR and tetrameric NA-RPARPAR (NA-R) for 5 min. (mean $\pm \mathrm{SEM}, \mathrm{N}=6$ independent experiments, the median from $n=4$ replicates per experiment was used for statistical analyses; Friedman test followed by Dunns' multiple comparison post hoc test; $\left.{ }^{*}, \mathrm{p}<0.05\right)$. 
Roth et al., Suppl. Fig. S7
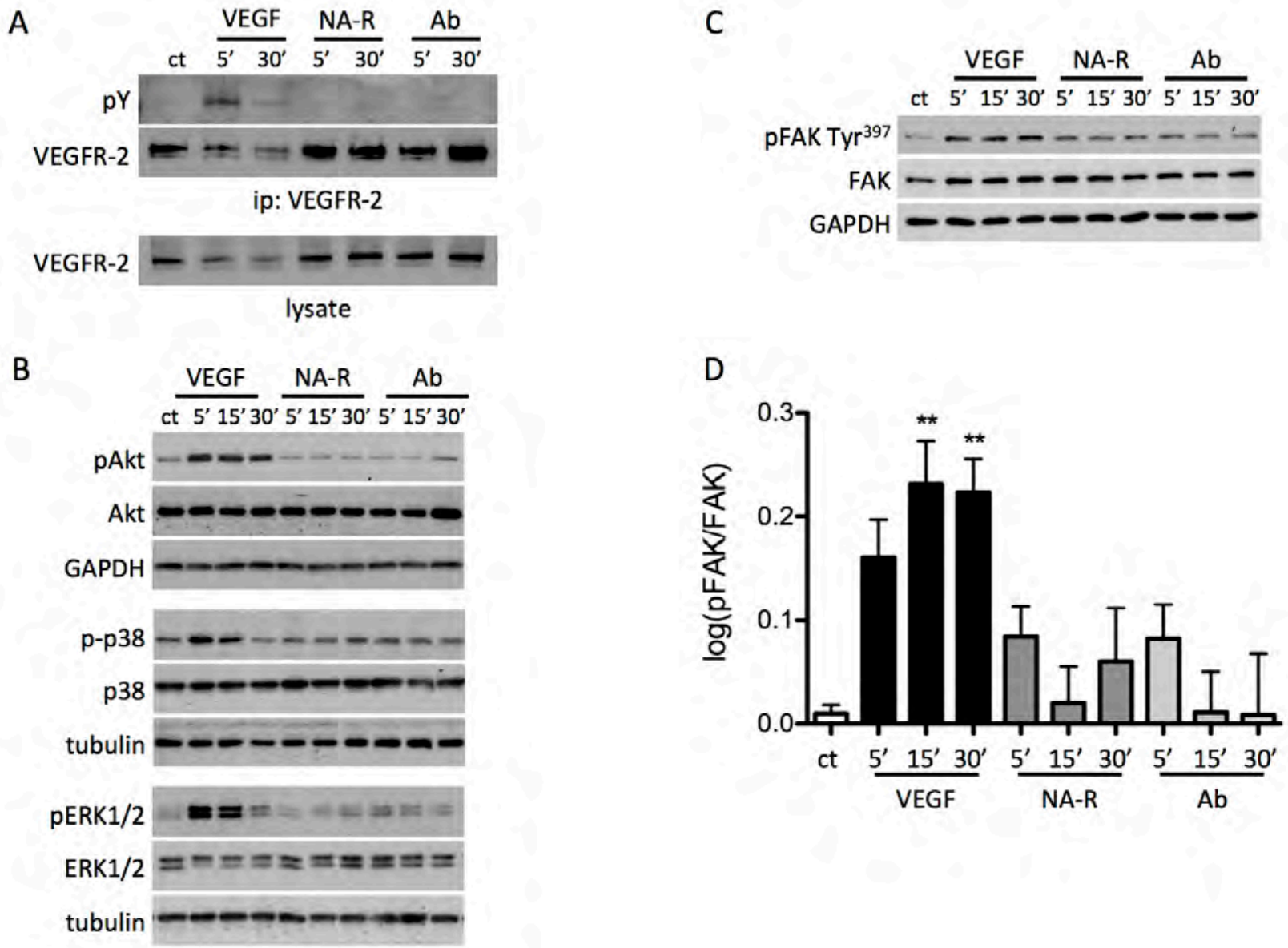

Figure S7. NA-RPARPAR and anti-NRP1 do not activate VEGFR-2, Akt, ERK1/2, p38, and FAK in HDBECs. (A-D) HDBEC monolayers were stimulated with VEGF-A 165 (VEGF), NA-RPARPAR (NA-R), or anti-NRP1 antibody (Ab). (A) VEGFR-2 immunoprecipitates were immunoblotted for anti-VEGFR-2 and phosphotyrosine, and total lysates were immunoblotted for anti-VEGFR-2. (B) Blotting was performed on total lysates with antibodies against phosphorylated Akt, ERK1/2, and p38, against the corresponding total proteins, and against the loading controls GAPDH and tubulin. Representative scans from $\mathrm{N}=3$ independent experiments. (C) Blotting was performed on total lysates with antibodies against phosphorylated FAK Tyr ${ }^{397}$, total FAK and the loading control GAPDH. Representative scans from $\mathrm{N}=4$ experiments. (D) The ratio pFAK:FAK was quantified using Fiji software (mean $\pm \mathrm{SEM} ; \mathrm{N}=4$ independent experiments; paired ANOVA test followed by Tukey's multiple comparison post hoc test; $\left.{ }^{* *}, \mathrm{p}<0.01\right)$. 
Roth et al., Suppl. Fig. S8

A

VEGF-A 165

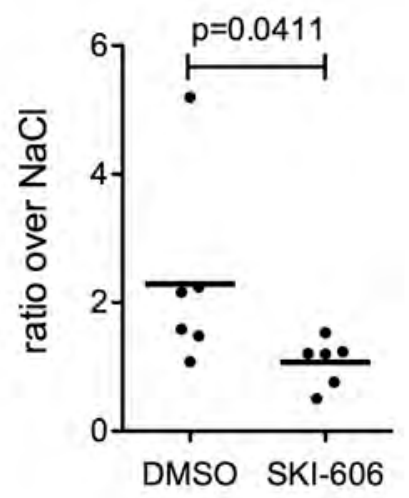

B

NA-RPARPAR

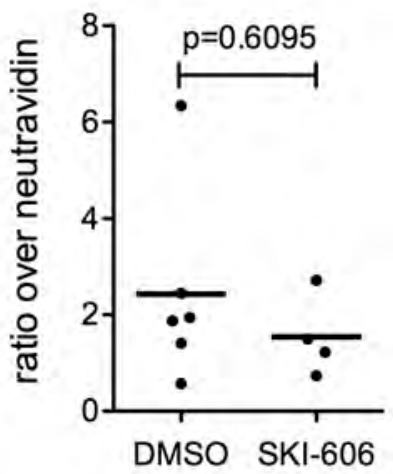

C

anti-NRP1

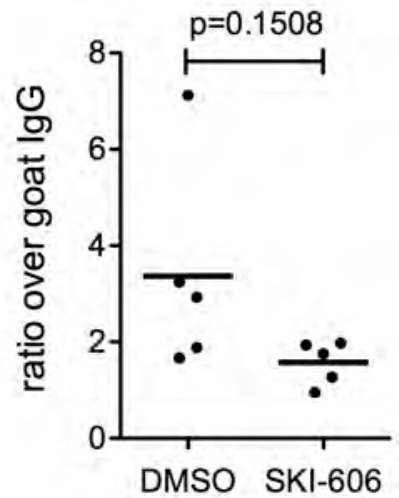

Figure S8. The Src inhibitor SKI-606 does not inhibit NA-RPARPAR- and anti-NRP1-induced vascular leakage. (A-C) Mice were injected intraperitoneally with DMSO or SKI-606 $5 \mathrm{mg} / \mathrm{kg}$ prior systemic treatment with Evans Blue. Leakage was induced with VEGF- $\mathrm{A}_{165}$ compared to $\mathrm{NaCl}$ (A), NA-RPARPAR compared to neutravidin (B), or anti-NRP1 compared to goat $\lg G(\mathbf{C})$, and results expressed as a ratio between the tested substance and its respective control (mean; N=4-6 mice per condition; MannWhitney test). 